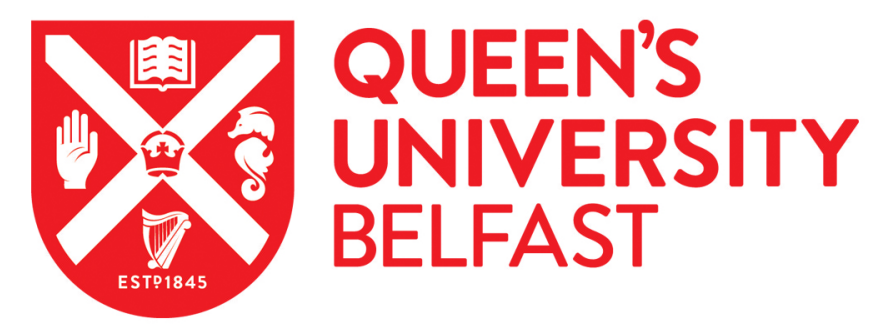

\title{
Improper Gaussian Signaling for Integrated Data and Energy Networking
}

Duong, T. Q., Yu, H., Tuan, H. D., Fang, Y., \& Hanzo, L. (2020). Improper Gaussian Signaling for Integrated Data and Energy Networking. IEEE Transactions on Communications.

https://doi.org/10.1109/TCOMM.2020.2981332

\section{Published in:}

IEEE Transactions on Communications

\section{Document Version:}

Peer reviewed version

\section{Queen's University Belfast - Research Portal:}

Link to publication record in Queen's University Belfast Research Portal

\section{Publisher rights}

(c) 2020 IEEE.

This work is made available online in accordance with the publisher's policies. Please refer to any applicable terms of use of the publisher.

\section{General rights}

Copyright for the publications made accessible via the Queen's University Belfast Research Portal is retained by the author(s) and / or other copyright owners and it is a condition of accessing these publications that users recognise and abide by the legal requirements associated with these rights.

Take down policy

The Research Portal is Queen's institutional repository that provides access to Queen's research output. Every effort has been made to ensure that content in the Research Portal does not infringe any person's rights, or applicable UK laws. If you discover content in the Research Portal that you believe breaches copyright or violates any law, please contact openaccess@qub.ac.uk. 


\title{
Improper Gaussian Signaling for Integrated Data and Energy Networking
}

\author{
H. Yu, H. D. Tuan, T. Q. Duong, Y. Fang, and L. Hanzo
}

\begin{abstract}
The paper considers the problem of beamforming design for a multi-cell network of downlink users, who either harvest energy or decode information or do both by receiving signals from the multi-antenna base station (BS) within a time slot and over the same frequency band. Our previous contributions have showed that the time-fraction based energy and information transmission, under which first the energy is transferred within the initial fraction of time and then the information is transferred within the remaining fraction, is the most efficient design alternative both in terms of its practical implementation and network performance. However, at the time of writing, both energy and information beamforming has only been implemented for proper Gaussian signaling (PGS), which has limited the network's throughput. Although the network throughput could be improved in some specific scenarios by using non-orthogonal multi-access (NOMA), this may compromise the user secrecy. In order to circumvent the above implementations, we conceive improper Gaussian signaling (IGS) for information beamforming, which enables the network to substantially improve its throughput in any scenario without jeopardizing the user secrecy despite its lowcomplexity signal processing at the user end. A simpler subclass of IGS is also considered, which also outperforms NOMA PGS and works under any arbitrary scenario.
\end{abstract}

Index Terms-Improper Gaussian signaling, multi-cell system, energy-harvesting, information throughput, nonconvex optimization, energy-harvesting constraint.

Hongwen $\mathrm{Yu}$ is the School of Communication and Information Engineering, Shanghai University, Shanghai, China (email: hongwenyu @ shu.edu.cn), and also with the School of Electrical and Data Engineering, University of Technology Sydney, Broadway, NSW 2007, Australia (email: hongwen.yu@student.uts.edu.au).

Hoang Duong Tuan is with the School of Electrical and Data Engineering, University of Technology Sydney, Broadway, NSW 2007, Australia (email: Tuan.Hoang@uts.edu.au).

Trung Q. Duong is with the School of Electronics, Electrical Engineering and Computer Science, Queen's University Belfast, Belfast BT7 1NN, UK (email: trung.q.duong@qub.ac.uk).

Yong Fang is with the School of Communication and Information Engineering, Shanghai University, Shanghai, China (email: yfang@ @staff.shu.edu.cn).

Lajos Hanzo with the School of Electronics and Computer Science, University of Southampton, Southampton, SO17 1BJ, U.K. (e-mail: 1h@ecs.soton.ac.uk).

H. D. Tuan would like to acknowledge the financial support of the Institute for Computational Science and Technology, Hochiminh City, Vietnam, and of the Australian Research Council's Discovery Projects under Project DP190102501.

T. Q. Duong would like to acknowledge the financial support of the U.K. Royal Academy of Engineering Research Fellowship under Grant RF1415 $\backslash 14 \backslash 22$.

Y. Fang would like to acknowledge the financial support of the National Natural Science Foundation of China (61673253).

L. Hanzo would like to acknowledge the financial support of the Engineering and Physical Sciences Research Council projects EP/Noo4558/1, EP/PO34284/1, and COALESCE, of the Royal Societys Global Challenges Research Fund Grant as well as of the European Research Council's Advanced Fellow Grant QuantCom

\section{INTRODUCTION}

The Internet-of-things (IoT) further broadens the challenges imposed on wireless communications by demanding wireless access for not only information but also for energy [1]. An access point may provide an information service or energy service, or both. In terms of base stations (BSs), it is expected that they are able to transfer not only information but also energy, requiring both high information throughput and substantial harvested energy. In fact, signal processing conceived for high information throughput aims for mitigating the interference at the receiver end, whilst interference actually can be beneficial for harvesting energy.

At the time of writing, there are two popular techniques of transfering information and energy over the same wireless medium within a time slot. The first one is the so-called simultaneous wireless information and power transfer (SWIPT) [2]-[6], which splits the received signal into two components, namely one for energy-harvesting (EH) and one for information decoding (ID) either by power splitting or time-switching (TS). Its practical implementation requires a sophisticated variable power-splitter [4]. From a signal processing perspective, it would be counterproductive to design a common beamformer to optimize the conflicting targets of information and energy beamforming at the same time. The second approach is the so-called time-fraction-based information and power transfer (TFIPT) relying on separate fractions of the time-slot [7]-[9], ${ }^{1}$ which may be conveniently implemented in practice and it is capable of outperforming SWIPT. Under this approach, the EH is improved by energy beamformers, while the information throughput is improved by information beamformers.

To improve the information throughput, which suffers from the network's ambition to provide EH service, we may invoke non-orthogonal multiple access (NOMA) (see e.g. [10], [11]), in order to compensate for the EH-induced throughput loss, when supporting multiple users. It was also shown in [11] that NOMA-based TFIPT outperforms its SWIPT counterpart. Since the main factor limiting the network throughput is multiuser interference, under NOMA the users of better channel conditions access and decode the information intended for users of poorer channel conditions to subtract it from their received signal before decoding their own information. However, this procedure degrades the secrecy of the users of poorer channel conditions. Moreover, the information throughput gain by NOMA is only substantial enough when the users channel conditions are strongly differentiated. Otherwise, conventional

\footnotetext{
${ }^{1}$ One should not confuse this with SWIPT, which splits the received signal using time-switching.
} 
orthogonal multi-access (OMA) is still preferred in terms of both its information throughput and user secrecy.

Proper Gaussian signaling (PGS) relying on circularly symmetric complex Gaussian (proper) signals is popular owing to its ease of analysis and design, but it requires the multi-user interference (MUI) to be completely suppressed [12]. This requirement may be eliminated by Improper Gaussian signaling (IGS) [13], [14], which was shown to exhibit supremacy over PGS in diverse practical scenarios, for example in single-input single-output (SISO) networks [15]-[22] or in MIMO interference networks [23]-[27] of multiple unicast transmitterreceiver pairs, as well as in broadcast networks [28]-[30] and in cognitive networks [31]-[33] relying on PGS for the primary users and IGS for the secondary users. Most recently, NOMA-PGS and NOMA-IGS was designed for multi-user multi-cell networks in [34]. In contrast to proper Gaussian signals having arbitrary covariance, improper Gaussian signals are characterized by the so-called augmented covariance of double size associated with a special structure involving its covariance and pseudo-covariance [13]. As such, in contrast to PGSs which are generated by linearly beamforming proper Gaussian sources, IGSs are generated by the widely linear beamforming of Gaussian sources, which are determined by a pair of correlated beamforming vectors. The design of beamforming vectors for IGS is more complex than for PGS not only because it involves twice the number of decision variables, but more importantly, the throughput functions are $\log$-determinant $\log \operatorname{det}($.$) even for multi-input single output$ (MISO) networks. Hence their optimization problem is much more computationally challenging than that the optimization of the logarithmic PGS throughput. However, as mentioned above, NOMA PGS requires additional processing at the users of better channel conditions to decode the information intended for the users of poorer channel conditions, and thus jeopardizes the secrecy of weaker users. By contrast, IGS improves the users' throughput without the above-mentioned extra NOMA-processing at the receiver end.

Against the above background, this paper proposes IGS for energy-harvesting aided networks with the following main contributions:

- We conceive and generate improper Gaussian signals by applying widely linear beamforming to proper Gaussian sources to improve the information throughput subject to EH constraints. The corresponding beamforming optimization problem becomes nonconvex, which involves $\log$ determinant functions, and thus it is computationally challenging. Hence a path-following computational procedure is proposed for this nonconvex problem, which iterates between improved feasible points and converges at least to a locally optimal solution.

- Additionally, we then develop a simplified IGS (s-IGS), which still improves the information throughput by applying linear beamforming to improper Gaussian sources. The resultant reduced-complexity beamforming optimization problem is then solved by a new path-following procedure.

- The simulation results provided demonstrate that both IGS and s-IGS outperform NOMA PGS. Hence,the in- formation throughput can be improved without any additional signal processing at the user end and yet the user secrecy is preserved.

The paper is organized as follows. Beamforming optimization problems for IGS and s-IGS are addressed in Sections II and Section III, respectively, while the simulations demonstrating their advantage over NOMA are provided in Section IV. Section V concludes the paper, which is followed by the Appendix. The Appendix develops new fundamental matrix inequalities, which were used for developing the path-following algorithms in Sections II and III.

Notation. Bold-faced lower-case (upper-case, resp.) letters, e.g. $\mathbf{x}$ (X, resp.), are used for vectors (matrices, resp.), and lower-case letters, e.g., $x$, are used for for scalars. The size of $N$-dimensional column vectors is $N \times 1$ while the size of $N$-dimensional row vectors is $1 \times N$. Analogously, the size of matrices with $N$ rows and $M$ columns is $N \times M$. $\mathbb{E}$ is the expectation operator. $\|.\|_{F}$ is the Frobenius norm of matrices. $[\mathbf{X}]^{2}$ is $\mathbf{X} \mathbf{X}^{H}$, and $\langle\mathbf{X}, \mathbf{Y}\rangle=\operatorname{trace}\left(\mathbf{X}^{H} \mathbf{Y}\right)$. The notation $\mathbf{X} \succ$ 0 ( $\mathbf{X} \succeq 0$, resp.) used for the Hermitian symmetric matrix $\mathbf{X}$ indicates that it is positive definite (positive semi-definite, resp.). $\mathbf{I}_{n}$ is the identity matrix of size $n \times n$. We also use $\langle\mathbf{A}\rangle=\operatorname{trace}(\mathbf{A}) . x_{\mathcal{S}}$ for the set $\mathcal{S}$ used as a subscription represents the set $\left\{x_{s}: s \in \mathcal{S}\right\}$, and $\mathbb{R}_{+}^{N} \triangleq\left\{\left(x_{1}, \ldots, x_{n}\right)^{T}\right.$ : $\left.x_{i}>0, i=1, \ldots, n\right\}$.

\section{SyStem MOdEL FOR IMPROPER GAUSSIAN SIGNAL PROCESSING}

Fig. 1 illustrates the downlink (DL) of a system consisting of $N$ cells under dense deployment, where the BS of each cell is equipped with $N_{t}$-transmit antennas (TAs) serving multiple single-antenna-aided users within its cell. In the $i$-th cell, there are $K$ energy-harvesting $(\mathrm{EH})$ users (EU) indexed by $\left(i, e_{1}\right), \ldots,\left(i, e_{K}\right)$, who harvest energy transferred by the BS through the wireless channels and thus have to be located sufficiently near to their BS. There are $M$ information-receiving users (IUs) indexed by $\left(i, d_{1}\right), \ldots,\left(i, d_{M}\right)$, who receive and decode information transmitted by the BS through the wireless DL channels. Note that there is a potential overlap between the sets of EUs and IUs, whenever there are users, who receive both energy and information from the BS through the same wireless channels. Then

$$
\mathcal{S}_{E} \triangleq\left\{\left(s, e_{\ell}\right): s=1, \ldots, N ; \ell=1, \ldots, K\right\}
$$

and

$$
\mathcal{S}_{I} \triangleq\left\{\left(s, d_{\ell}\right): s=1, \ldots, N ; \ell=1, \ldots, M\right\}
$$

respectively represent the set of EUs and IUs. Under timefraction-based information and energy transfer [7]-[9], the specific fraction of time $0<1 / t_{1}<1$ is used for power transfer, while the remaining fraction of $0<1 / t_{2}<1$ is used for information transfer. Let $\mathbf{h}_{s, i, e_{j}} \in \mathbb{C}^{1 \times N_{t}}$ be the channel spanning from the $\mathrm{BS} s$ to the $\operatorname{EU}\left(i, e_{j}\right), \mathbf{x}_{s, e_{\ell}}^{E}=\mathbf{v}_{s, e_{\ell}} \tilde{s}_{s, e_{\ell}} \in$ $\mathbb{C}^{N_{t} \times 1}$ be the beamformed energy signal intended for the EU $\left(s, e_{\ell}\right)$, where $\mathbf{v}_{s, e_{\ell}} \in \mathbb{C}^{N_{t} \times 1}$ is the energy beamformer and $\tilde{s}_{s, e_{\ell}} \in \mathbb{C}$ is the energy symbol with $\mathbb{E}\left(\left|\tilde{s}_{s, e_{\ell}}\right|^{2}\right)=1$. All 


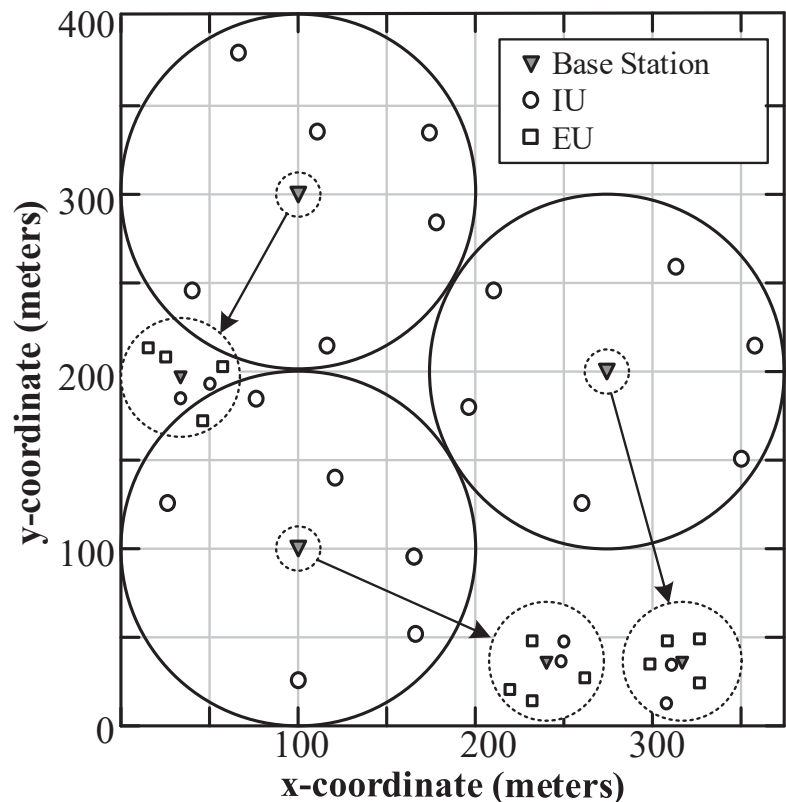

Fig. 1: Multi-cell energy-harvesting aided system

$\tilde{s}_{s, e_{\ell}}, s=1, \ldots, N ; \ell=1, \ldots, K$ are independent. The signal received by the $\mathrm{EU}\left(i, e_{j}\right)$ is

$$
y_{i, e_{j}}=\sum_{\left(s, e_{\ell}\right) \in \mathcal{S}_{E}} \mathbf{h}_{s, i, e_{j}} \mathbf{x}_{s, e_{\ell}}^{E}+n_{i, e_{j}},
$$

where $n_{i, e_{j}} \in \mathbb{C}$ is the background noise, which is proper Gaussian with zero mean and variance $\sigma^{2}$. Given the energy conversion efficiency $0<\zeta<1$, the energy harvested by the $\mathrm{EU}\left(i, e_{j}\right) \in \mathcal{S}_{E}$ is $\left(1 / t_{1}\right) \zeta \mathbb{E}\left(\left|y_{i, e_{j}}\right|^{2}\right)$, which is ${ }^{2}$

$$
\left(1 / t_{1}\right) \zeta \pi_{i, e_{j}}\left(\mathbf{v}_{\mathcal{S}_{E}}\right),
$$

where

$$
\pi_{i, e_{j}}\left(\mathbf{v}_{\mathcal{S}_{E}}\right)=\sum_{\ell=1}^{K}\left|\mathbf{h}_{i, i, e_{j}} \mathbf{v}_{i, e_{\ell}}\right|^{2} .
$$

In (3), the energy of the background noise $n_{i, e_{j}}$ and the interference from the BSs of the other cells are low for $\mathrm{EH}$ and thus are ignored.

Let $n_{i, d_{j}} \in \mathbb{C}$ be the background noise at IU $\left(i, d_{j}\right)$, which is proper Gaussian with zero mean and variance $\sigma^{2}$. For the information transfer during the remaining $1 / t_{2}$ fraction of time, the signal received by the IU $\left(i, d_{j}\right)$ is

$$
y_{i, d_{j}}=\sum_{\left(s, d_{\ell}\right) \in \mathcal{S}_{I}} \mathbf{h}_{s, i, d_{j}} \mathbf{x}_{s, d_{\ell}}^{\mathbf{I}}+n_{i, d_{j}},
$$

where in general, the beamformed information signal intended for the IU $\left(s, d_{\ell}\right)$ is denoted as $\mathbf{x}_{s, d_{\ell}}^{\mathbf{I}} \in \mathbb{C}^{N_{t} \times 1}$ in (4), which is improper Gaussian, i.e. $\mathbb{E}\left(\left(\mathbf{x}_{s, d_{\ell}}^{\mathbf{I}}\right)^{2}\right) \neq 0$, and it is generated by widely linear beamforming of a normalized proper

\footnotetext{
${ }^{2}$ The RF-to-harvested energy conversion function is in practice non-linear. However, there is no generally agreed accurate function at the time of writing. Hence, to avoid obfuscating the salient IGS-related trends, we have opted for this simple linear model.
}

Gaussian source $s_{s, d_{\ell}}\left(\mathbb{E}\left(\left|s_{s, d_{\ell}}\right|^{2}\right)=1\right.$ and $\left.\mathbb{E}\left(\left(s_{s, d_{\ell}}\right)^{2}\right)=0\right)$ as [34]

$$
\mathbf{x}_{s, d_{\ell}}^{\mathbf{I}}=\boldsymbol{w}_{1, s, d_{\ell}} s_{s, d_{\ell}}+\boldsymbol{w}_{2, s, d_{\ell}} s_{s, d_{\ell}}^{*}
$$

with the aid of the beamformers $\boldsymbol{w}_{1, s, d_{\ell}} \in \mathbb{C}^{N_{t} \times 1}$ and $\boldsymbol{w}_{2, s, d_{\ell}} \in \mathbb{C}^{N_{t} \times 1}$. Then, the signal received in (4) at the IU $\left(i, d_{j}\right)$ is rewritten as

$$
y_{i, d_{j}}=\sum_{\left(s, d_{\ell}\right) \in \mathcal{S}_{I}} \mathbf{h}_{s, i, d_{j}}\left(\boldsymbol{w}_{1, s, d_{\ell}} s_{s, d_{\ell}}+\boldsymbol{w}_{2, s, d_{\ell}} s_{s, d_{\ell}}^{*}\right)+n_{i, d_{j}} .
$$

By writing

$$
\left(\mathbf{x}_{s, d_{\ell}}^{\mathbf{I}}\right)^{*}=\left[\begin{array}{ll}
\boldsymbol{w}_{2, s, d_{\ell}}^{*} & \boldsymbol{w}_{1, s, d_{\ell}}^{*}
\end{array}\right]\left[\begin{array}{l}
s_{s, d_{\ell}} \\
s_{s, d_{\ell}}^{*}
\end{array}\right]
$$

and defining $\boldsymbol{w}_{s, d_{\ell}}=\left\{\boldsymbol{w}_{j, s, d_{\ell}}, j=1,2\right\}$, we can express the augmented equation of (6) as

$$
\begin{aligned}
\overline{\mathbf{y}}_{i, d_{j}} \triangleq & {\left[\begin{array}{l}
y_{i, d_{j}} \\
y_{i, d_{j}}^{*}
\end{array}\right] } \\
= & \sum_{\left(s, d_{\ell}\right) \in \mathcal{S}_{I}}\left[\begin{array}{ll}
\mathbf{h}_{s, i, d_{j}} \boldsymbol{w}_{1, s, d_{\ell}} & \mathbf{h}_{s, i, d_{j}} \boldsymbol{w}_{2, s, d_{\ell}} \\
\mathbf{h}_{s, i, d_{j}}^{*} \boldsymbol{w}_{2, s, d_{\ell}}^{*} & \mathbf{h}_{s, i, d_{j}}^{*} \boldsymbol{w}_{1, s, d_{\ell}}^{*}
\end{array}\right]\left[\begin{array}{l}
s_{s, d_{\ell}} \\
s_{s, d_{\ell}}^{*}
\end{array}\right] \\
& +\left[\begin{array}{l}
n_{i, d_{j}} \\
n_{i, d_{j}}^{*}
\end{array}\right] \\
= & \Lambda_{i, i, d_{j}}\left(\boldsymbol{w}_{i, d_{j}}\right) \overline{\mathbf{s}}_{i, d_{j}} \\
& +\sum_{\left(s, d_{\ell}\right) \in \mathcal{S}_{I} \backslash\left\{i, d_{j}\right\}} \Lambda_{s, i, d_{j}}\left(\boldsymbol{w}_{s, d_{\ell}}\right) \overline{\mathbf{s}}_{s, d_{\ell}}+\overline{\mathbf{n}}_{i, d_{j}}
\end{aligned}
$$

for

$$
\Lambda_{s, i, d_{j}}\left(\boldsymbol{w}_{s, d_{\ell}}\right) \triangleq\left[\begin{array}{ll}
\mathbf{h}_{s, i, d_{j}} \boldsymbol{w}_{1, s, d_{\ell}} & \mathbf{h}_{s, i, d_{j}} \boldsymbol{w}_{2, s, d_{\ell}} \\
\mathbf{h}_{s, i, d_{j}}^{*} \boldsymbol{w}_{2, s, d_{\ell}}^{*} & \mathbf{h}_{s, i, d_{j}}^{*} \boldsymbol{w}_{1, s, d_{\ell}}^{*}
\end{array}\right],
$$

which represents a linear mapping from $\mathbb{C}^{\left(2 N_{t}\right) \times 1}$ to $\mathbb{C}^{2 \times 2}$, and

$$
\overline{\mathbf{s}}_{s, d_{\ell}} \triangleq\left[\begin{array}{l}
s_{s, d_{\ell}} \\
s_{s, d_{\ell}}^{*}
\end{array}\right] \in \mathbb{C}^{2}, \overline{\mathbf{n}}_{i, d_{j}} \triangleq\left[\begin{array}{l}
n_{i, d_{j}} \\
n_{i, d_{j}}^{*}
\end{array}\right] \in \mathbb{C}^{2}
$$

It may be readily shown that

$$
\mathbb{E}\left\{\left[\overline{\mathbf{s}}_{i, d_{\ell}}\right]^{2}\right\}=\mathbf{I}_{2}, \mathbb{E}\left\{\left[\overline{\mathbf{n}}_{i, d_{j}}\right]^{2}\right\}=\sigma^{2} \mathbf{I}_{2},
$$

and

$$
\mathbb{E}\left\{\left[\Lambda_{s, i, d_{j}}\left(\boldsymbol{w}_{s, d_{\ell}}\right) \overline{\mathbf{s}}_{i, d_{j}}\right]^{2}\right\}=\left[\Lambda_{s, i, d_{j}}\left(\boldsymbol{w}_{s, d_{\ell}}\right)\right]^{2} .
$$

The throughput at the IU $\left(i, d_{j}\right)$ expressed in nats/sec/Hz is given by the mutual information (MI) between $\bar{y}_{i, d_{j}}$ and $\bar{s}_{i, d_{j}}$ computed as [35]

$$
\frac{1}{2 t_{2}} r_{i, d_{j}}\left(\boldsymbol{w}_{\mathcal{S}_{I}}\right)
$$

for

$r_{i, d_{j}}\left(\boldsymbol{w}_{\mathcal{S}_{I}}\right)=\ln \left|\mathbf{I}_{2}+\left[\Lambda_{i, i, d_{j}}\left(\boldsymbol{w}_{i, d_{j}}\right)\right]^{2}\left(\Psi_{i, d_{j}}\left(\boldsymbol{w}_{\mathcal{S}_{I}}\right)\right)^{-1}\right|$,

and

$$
\Psi_{i, d_{j}}\left(\boldsymbol{w}_{\mathcal{S}_{I}}\right) \triangleq \sum_{\left(s, d_{\ell}\right) \in \mathcal{S}_{I} \backslash\left\{\left(i, d_{j}\right)\right\}}\left[\Lambda_{s, i, d_{j}}\left(\boldsymbol{w}_{s, d_{\ell}}\right)\right]^{2}+\sigma^{2} \mathbf{I}_{2} .
$$


Based on (2) and (12), we consider the following problem of max-min throughput optimization

$$
\begin{array}{r}
\max _{\mathbf{v}_{\mathcal{S}_{E}}, \boldsymbol{w}_{\mathcal{S}_{I}}, \mathbf{t}=\left(t_{1}, t_{2}\right)^{T} \in \mathbb{R}_{+}^{2}, \gamma} \gamma \quad \text { s.t. (15a) } \\
r_{i, d_{j}}\left(\boldsymbol{w}_{\mathcal{S}_{I}}\right) \geq 2 \gamma t_{2},\left(i, d_{j}\right) \in \mathcal{S}_{I},(15 \mathrm{~b}) \\
\pi_{i, e_{j}}\left(\mathbf{v}_{\mathcal{S}_{E}}\right) \geq \frac{e_{\min }}{\zeta} t_{1},\left(i, e_{j}\right) \in \mathcal{S}_{E},(15 \mathrm{c}) \\
\frac{1}{t_{1}}+\frac{1}{t_{2}} \leq 1,(15 \mathrm{~d}) \\
\frac{1}{t_{1}} \sum_{j=1}^{K}\left\|\mathbf{v}_{i, e_{j}}\right\|^{2}+\frac{\sum_{j=1}^{M}\left\|\boldsymbol{w}_{i, d_{j}}\right\|^{2}}{t_{2}} \leq P, i=1, \ldots, N,(15 \mathrm{e}) \\
\left\|\mathbf{v}_{i, e_{j}}\right\|^{2} \leq P,\left(i, e_{j}\right) \in \mathcal{S}_{E} ;\left\|\boldsymbol{w}_{i, d_{j}}\right\|^{2} \leq P,\left(i, d_{j}\right) \in \mathcal{S}_{I},(15 \mathrm{f})
\end{array}
$$

where $P$ is the power budget granted for each BS and we have

$$
\left\|\boldsymbol{w}_{i, d_{j}}\right\|^{2}=\left\|\boldsymbol{w}_{1, i, d_{j}}\right\|^{2}+\left\|\boldsymbol{w}_{2, i, d_{j}}\right\|^{2},
$$

which is the power of the widely linear beamformer in (5). Note that by (15b), $\gamma$ in (15a) represents $\min _{\left(i, d_{j}\right) \in \mathcal{S}_{I}}\left(1 / 2 t_{2}\right) r_{i, d_{j}}\left(\boldsymbol{w}_{\mathcal{S}_{I}}\right)$, i.e. it is the minimal value among the IUs' throughput $\left(1 / 2 t_{2}\right) r_{i, d_{j}}\left(\boldsymbol{w}_{\mathcal{S}_{I}}\right),\left(i, d_{j}\right) \in \mathcal{S}_{I}$. Furthermore, $e_{\min }$ in $(15 \mathrm{c})$ is the threshold of $\mathrm{EH}$, so the constraint (15c) sets the threshold in serving the EUs. The constraint (15d) restricts the fractional-time-based implementation within a single time slot, and (15e) is a typical sum-power constraint, while (15f) is the physical transmission power constraint. The last three constraints (15d)-(15f) in (15) are convex.

The problem (15) is nonconvex because the pair of constraints (15b) and (15c) representing the information throughput and EH power are nonconvex. To develop a path-following algorithm for its computation, which improves its feasible value in each iteration, we have to develop an inner convex approximation of these nonconvex constraints.

Let $\left(\mathbf{v}_{\mathcal{S}_{E}}^{(\kappa)}, \mathbf{w}_{\mathcal{S}_{I}}^{(\kappa)}, \mathbf{t}^{(\kappa)}, \gamma^{(\kappa)}\right)$ be the feasible point for (15) found from the $(\kappa-1)$ th iteration.

To obtain an inner convex approximation of (15b) we have to derive a concave upper bounding function approximation of its left-hand-side (LHS) and a convex lower bounding function approximation of its right-hand-side (RHS). Applying the inequality (60) in the Appendix yields the following concave upper bounding approximation of the LHS of (15b):

$$
\begin{aligned}
r_{i, d_{j}}\left(\boldsymbol{w}_{\mathcal{S}_{I}}\right) \geq & a_{i, d_{j}}^{(\kappa)}+2\left\langle\mathbf{B}_{i, d_{j}}^{(\kappa)} \Lambda_{i, i, d_{j}}\left(\boldsymbol{w}_{i, d_{j}}\right)\right\rangle \\
& -\left\langle\mathbf{C}_{i, d_{j}}^{(\kappa)},\left[\Lambda_{i, i, d_{j}}\left(\boldsymbol{w}_{i, d_{j}}\right)\right]^{2}+\Psi_{i, d_{j}}\left(\boldsymbol{w}_{\mathcal{S}_{I}}\right)\right\rangle \\
= & a_{i, d_{j}}^{(\kappa)}-\sigma^{2}\left\langle\mathbf{C}_{i, d_{j}}^{(\kappa)}\right\rangle+2\left\langle\mathbf{B}_{i, d_{j}}^{(\kappa)} \Lambda_{i, i, d_{j}}\left(\boldsymbol{w}_{i, d_{j}}\right)\right\rangle \\
& -\left\langle\mathbf{C}_{i, d_{j}}^{(\kappa)}, \sum_{\left(s, d_{\ell}\right) \in \mathcal{S}_{I}}\left[\Lambda_{s, i, d_{j}}\left(\boldsymbol{w}_{s, d_{\ell}}\right)\right]^{2}\right\rangle \\
= & a_{i, d_{j}}^{(\kappa)}-\sigma^{2}\left\langle\mathbf{C}_{i, d_{j}}^{(\kappa)}\right\rangle+2\left\langle\mathbf{B}_{i, d_{j}}^{(\kappa)} \Lambda_{i, i, d_{j}}\left(\boldsymbol{w}_{i, d_{j}}\right)\right\rangle \\
& -\sum_{\left(s, d_{\ell}\right) \in \mathcal{S}_{I}}\left\langle\Lambda_{s, i, d_{j}}^{H}\left(\boldsymbol{w}_{s, d_{\ell}}\right) \mathbf{C}_{i, d_{j}}^{(\kappa)} \Lambda_{s, i, d_{j}}\left(\boldsymbol{w}_{s, d_{\ell}}\right)\right\rangle \\
= & a_{i, d_{j}}^{(\kappa)}-\sigma^{2}\left\langle\mathbf{C}_{i, d_{j}}^{(\kappa)}\right\rangle+2\left\langle\mathbf{B}_{i, d_{j}}^{(\kappa)} \Lambda_{i, i, d_{j}}\left(\boldsymbol{w}_{i, d_{j}}\right)\right\rangle \\
& -\sum_{\left(s, d_{\ell}\right) \in \mathcal{S}}\left\|\left(\mathbf{C}_{i, d_{j}}^{(\kappa)}\right)^{1 / 2} \Lambda_{s, i, d_{j}}\left(\boldsymbol{w}_{s, d_{\ell}}\right)\right\|_{F}^{2} \\
\triangleq & r_{i, d_{j}}^{(\kappa)}\left(\boldsymbol{w}_{\mathcal{S}_{I}}\right),
\end{aligned}
$$

where we have

$$
\begin{aligned}
a_{i, d_{j}}^{(\kappa)}= & r_{i, d_{j}}\left(\mathbf{w}_{\mathcal{S}_{I}}^{(\kappa)}\right) \\
& -\left\langle\left[\Lambda_{i, i, d_{j}}\left(\mathbf{w}_{i, d_{j}}^{(\kappa)}\right)\right]^{2}\left(\Psi_{i, d_{j}}\left(\mathbf{w}_{\mathcal{S}_{I}}^{(\kappa)}\right)\right)^{-1} \chi_{(1} 17\right) \\
\mathbf{B}_{i, d_{j}}^{(\kappa)}= & \left(\Lambda_{i, i, d_{j}}\left(\mathbf{w}_{i, d_{j}}^{(\kappa)}\right)\right)^{H}\left(\Psi_{i, d_{j}}\left(\mathbf{w}_{\mathcal{S}_{I}}^{(\kappa)}\right)\right)^{-1},(17 b) \\
0 \mathbf{C}_{i, d_{j}}^{(\kappa)}= & \left(\Psi_{i, d_{j}}\left(\mathbf{w}_{\mathcal{S}_{I}}^{(\kappa)}\right)\right)^{-1} \\
& -\left(\left[\Lambda_{i, i, d_{j}}\left(\mathbf{w}_{i, d_{j}}^{(\kappa)}\right)\right]^{2}+\Psi_{i, d_{j}}\left(\mathbf{w}_{\mathcal{S}_{I}}^{(\kappa)}\right)\right)^{-1}
\end{aligned}
$$

Meanwhile, the RHS of (15b) is upper bounded as follows:

$$
2 \gamma t_{2} \leq \frac{\gamma^{(\kappa)} t_{2}^{(\kappa)}}{2}\left(\frac{\gamma}{\gamma^{(\kappa)}}+\frac{t_{2}}{t_{2}^{(\kappa)}}\right)^{2}
$$

Using (16) and (18), the nonconvex constraint (15b) is innerly approximated by the following convex constraint in the sense that any feasible point for the latter is also feasible for the former:

$$
r_{i, d_{j}}^{(\kappa)}\left(\boldsymbol{w}_{\mathcal{S}}\right) \geq \frac{\gamma^{(\kappa)} t_{2}^{(\kappa)}}{2}\left(\frac{\gamma}{\gamma^{(\kappa)}}+\frac{t_{2}}{t_{2}^{(\kappa)}}\right)^{2},\left(i, d_{j}\right) \in \mathcal{S}_{I} .
$$

From (3), the LHS of (15c) is seen to be convex quadratic, hence the nonconvex constraint (15c) is said to be reverse convex and can be innerly approximated by a convex constraint by linearizing its LHS at $\mathbf{v}_{\mathcal{S}_{e}}^{(\kappa)}$ [36]

$$
\begin{aligned}
\sum_{\ell=1}^{K}[2 \Re & \left\{\left(\mathbf{v}_{i, e_{\ell}}^{(\kappa)}\right)^{H} \mathbf{h}_{i, i, e_{j}}^{H} \mathbf{h}_{i, i, e_{j}} \mathbf{v}_{i, e_{\ell}}\right\} \\
& \left.-\left|\mathbf{h}_{i, i, e_{j}} \mathbf{v}_{i, e_{\ell}}^{(\kappa)}\right|^{2}\right] \geq \frac{e_{\min }}{\zeta} t_{1}, \quad\left(i, e_{j}\right) \in \mathcal{S}_{E},
\end{aligned}
$$

which was used in the previous treatises of [7]-[9] handling EH constraints.

At the $\kappa$ th iteration we solve the following convex problem, which provides a feasible value for (15), to generate the next feasible point $\left(\mathbf{v}_{\mathcal{S}_{E}}^{(\kappa+1)}, \mathbf{w}_{\mathcal{S}_{I}}^{(\kappa+1)}, \mathbf{t}^{(\kappa+1)}, \gamma^{(\kappa+1)}\right)$ for (15):

$$
\max _{\mathbf{v}_{\mathcal{S}_{E}}, \boldsymbol{w}_{\mathcal{S}_{I}}, \mathbf{t}=\left(t_{1}, t_{2}\right)^{T} \in \mathbb{R}_{+}^{2}, \gamma} \gamma \text { s.t. } \quad(15 d)-(15 f),(19),(20) \text {. }
$$

This convex problem involves $n_{v}=N N_{t}(K+2 M)+3$ decision variables and $m_{c}=1+N(K+M+1)$ quadratic constraints, hence its computational complexity is [37]

$$
\mathcal{O}\left(m_{c}^{2.5}\left(n_{v}^{2}+m_{c}\right)\right)
$$

Note that we have $\gamma^{(\kappa+1)}>\gamma^{(\kappa)}$ as long as $\left(\mathbf{v}_{\mathcal{S}_{E}}^{(\kappa+1)}, \mathbf{w}_{\mathcal{S}_{I}}^{(\kappa+1)}, \mathbf{t}^{(\kappa+1)}, \gamma^{(\kappa+1)}\right) \neq\left(\mathbf{v}_{\mathcal{S}_{E}}^{(\kappa)}, \mathbf{w}_{\mathcal{S}_{I}}^{(\kappa)}, \mathbf{t}^{(\kappa)}, \gamma^{(\kappa)}\right)$, because they respectively are the optimal solution and a feasible point for (21). This means that $\left(\mathbf{v}_{\mathcal{S}_{E}}^{(\kappa+1)}, \mathbf{w}_{\mathcal{S}_{I}}^{(\kappa+1)}, \mathbf{t}^{(\kappa+1)}, \gamma^{(\kappa+1)}\right)$ is a better feasible point than $\left(\mathbf{v}_{\mathcal{S}_{E}}^{(\kappa)}, \mathbf{w}_{\mathcal{S}_{I}}^{(\kappa)}, \mathbf{t}^{(\kappa)}, \gamma^{(\kappa)}\right)$ for (15). As such, the sequence $\left\{\left(\mathbf{v}_{\mathcal{S}_{E}}^{(\kappa)}, \mathbf{w}_{\mathcal{S}_{I}}^{(\kappa)}, t^{(\kappa)}, \gamma^{(\kappa)}\right)\right\}$ of feasible points for (15) converges at least to a point satisfying the Karush-Kuh-Tucker (KKT) condition of optimality [38]. Our previous result (see e.g. [9]) shows that such a point often turns out to be the globally optimal solution of (15). 
It is important to locate a feasible point $\left(\mathbf{v}_{\mathcal{S}_{E}}^{(0)}, \boldsymbol{w}_{\mathcal{S}_{I}}^{(0)}, \mathbf{t}^{(0)}, \gamma^{(0)}\right)$ for (15) for initializing the pathfollowing procedure. Let us fix $\mathbf{t}^{(0)}=\left(t_{1}^{(0)}, t_{2}^{(0)}\right)$ and good $\gamma^{(0)}$ and randomly generate $\left(\mathbf{v}_{\mathcal{S}_{E}}^{(0)}, \boldsymbol{w}_{\mathcal{S}_{I}}^{(0)}\right)$ feasible for (15e)-(15f). Then iterate as follows

$$
\begin{aligned}
& \max _{\mathbf{v}_{\mathcal{S}_{E}}, \boldsymbol{w}_{\mathcal{S}_{I}, \eta}} \eta \quad \text { s.t. } \quad(15 f) \\
& r_{i, d_{j}}^{(\kappa)}\left(\boldsymbol{w}_{\mathcal{S}_{I}}\right) \geq 2 \gamma^{(0)} t_{2}^{(0)} \eta,\left(i, d_{j}\right) \in \mathcal{S}_{I}, \\
& \sum_{\ell=1}^{K}\left[2 \Re\left\{\left(\mathbf{v}_{i, e_{\ell}}^{(\kappa)}\right)^{H} \mathbf{h}_{i, i, e_{j}} \mathbf{h}_{i, i, e_{j}}^{H} \mathbf{v}_{i, e_{\ell}}\right\}-\left|\mathbf{h}_{i, i, e_{j}}^{H} \mathbf{v}_{i, e_{\ell}}^{(\kappa)}\right|^{2}\right] \\
& \geq \frac{e_{\min }}{\zeta} t_{1}^{(0)} \eta,\left(i, e_{j}\right) \in \mathcal{S}_{E}, \\
& \frac{1}{t_{1}^{(0)}} \sum_{j=1}^{K}\left\|\mathbf{v}_{i, e_{j}}\right\|^{2}+\frac{\sum_{j=1}^{M}\left\|\boldsymbol{w}_{i, d_{j}}\right\|^{2}}{2 t_{2}^{(0)}} \leq P, \\
& i=1, \ldots, N,
\end{aligned}
$$

until reaching $\eta \geq 1$ at $\left(\mathbf{v}_{\mathcal{S}_{E}}^{(0)}, \boldsymbol{w}_{\mathcal{S}_{I}}^{(0)}\right)$ in order to guarantee that $\left(\mathbf{t}^{(0)}, \gamma^{(0)} \eta, \boldsymbol{w}_{\mathcal{S}_{I}}^{(\kappa)}, \mathbf{v}_{\mathcal{S}_{E}}^{(\kappa)}\right)$ is feasible for (15).

Algorithm 1 represents the formal pseudo code of the above computational procedure.

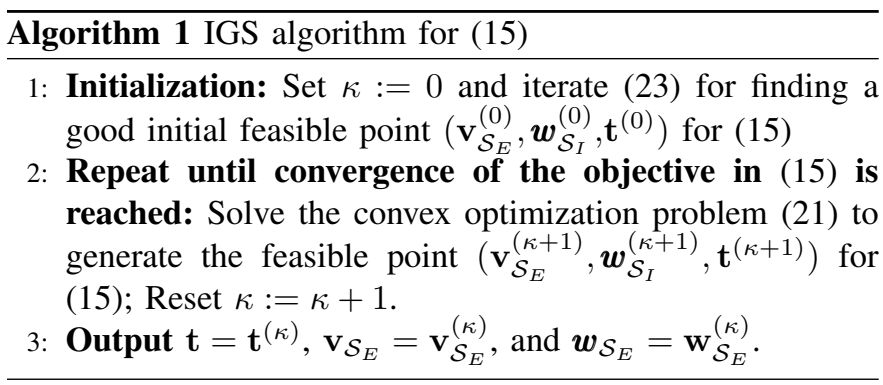

\section{SIMPLIFIED IMPROPER GAUSSIAN SIGNALING}

In (5), the improper Gaussian signal $\mathbf{x}_{s, d_{\ell}}^{\mathbf{I}}$ is generated as a widely linear transform of a proper Gaussian source $s_{s, d_{\ell}}$. By contrast, in this section, the improper Gaussian signal $\mathbf{x}_{s, d_{\ell}}^{\mathbf{I}}$ in (4) is generated as a linear transform of an improper Gaussian source as follows

$$
\mathbf{x}_{s, d_{\ell}}^{\mathbf{I}}=\boldsymbol{w}_{s, d_{\ell}} s_{s, d_{\ell}}, \boldsymbol{w}_{s, d_{\ell}} \in \mathbb{C}^{N_{t} \times 1},
$$

where $s_{s, d_{\ell}}$ is a normalized improper Gaussian random variable $\left(\mathbb{E}\left(\left|s_{s, d_{\ell}}\right|^{2}\right)=1\right)$, which is fully characterized by the augmented covariance defined in [13]:

$$
\begin{aligned}
\mathbf{P}_{s, d_{\ell}} & =\left[\begin{array}{ll}
\mathbb{E}\left(\left|s_{s, d_{\ell}}\right|^{2}\right) & \mathbb{E}\left(s_{s, d_{\ell}}^{2}\right) \\
\left(\mathbb{E}\left(s_{s, d_{\ell}}^{2}\right)\right)^{*} & \mathbb{E}\left(\left|s_{s, d_{\ell}}\right|^{2}\right)
\end{array}\right] \\
& =\mathbb{E}\left\{\left[\begin{array}{l}
s_{s, d_{\ell}} \\
s_{s, d_{\ell}}^{*}
\end{array}\right]\left[\begin{array}{l}
s_{s, d_{\ell}} \\
s_{s, d_{\ell}}^{*}
\end{array}\right]^{H}\right\} \\
& =\left[\begin{array}{cc}
1 & q_{s, d_{\ell}} \\
q_{s, d_{\ell}}^{*} & 1
\end{array}\right]
\end{aligned}
$$

with $q_{s, d_{\ell}} \in \mathbb{C}$ satisfying the following convex quadratic constraint to make it qualified as a pseudo-covariance of $s_{s, d_{\ell}}$

$$
\left|q_{s, d_{\ell}}\right|^{2}<1,\left(s, d_{\ell}\right) \in \mathcal{S}_{I}
$$

which makes $\mathbf{P}_{s, d_{\ell}}$ positive definite. Note that $q_{s, d_{\ell}}=0$ in (25) means $\mathbb{E}\left(s_{s, d_{\ell}}^{2}\right)=0$, i.e. $s_{s, d_{\ell}}$ becomes proper.

By taking the square root according to

$$
\mathbf{P}_{s, d_{\ell}}^{1 / 2}=\left[\begin{array}{ll}
\alpha_{s, d_{\ell}} & \beta_{s, d_{\ell}} \\
\beta_{s, d_{\ell}}^{*} & \alpha_{s, d_{\ell}}
\end{array}\right] \succeq 0
$$

in conjunction with

$$
\begin{gathered}
\alpha_{s, d_{\ell}}=\frac{\left(1+\sqrt{1-\mid q_{s,\left.d_{\ell}\right|^{2}}}\right)^{1 / 2}}{\sqrt{2}}, \\
\beta_{s, d_{\ell}}=\frac{q_{s, d_{\ell}}}{\sqrt{2}\left(1+\sqrt{1-\left|q_{s, d_{\ell}}\right|^{2}}\right)^{1 / 2}},
\end{gathered}
$$

it can be readily shown that

$$
s_{s, d_{\ell}}=\alpha_{s, d_{\ell}} \tilde{s}_{s, d_{\ell}}+\beta_{s, d_{\ell}} \tilde{s}_{s, d_{\ell}}^{*}
$$

for a normalized proper Gaussian $\tilde{s}_{s, d_{\ell}}$. Therefore (24) can be written in the widely linear form

$$
\mathbf{x}_{s, d_{\ell}}^{\mathbf{I}}=\boldsymbol{w}_{s, d_{\ell}} \alpha_{s, d_{\ell}} \tilde{s}_{s, d_{\ell}}+\boldsymbol{w}_{s, d_{\ell}} \beta_{s, d_{\ell}} \tilde{s}_{s, d_{\ell}}^{*},
$$

which a particular class of (5) associated with

$$
\boldsymbol{w}_{1, s, d_{\ell}}=\alpha_{s, d_{\ell}} \boldsymbol{w}_{s, d_{\ell}} \quad \& \quad \boldsymbol{w}_{2, s, d_{\ell}}=\beta_{s, d_{\ell}} \boldsymbol{w}_{s, d_{\ell}} .
$$

The advantage of (24) over (5) is that for each $\left(s, d_{\ell}\right)$, the former involves only $N_{t}+1$ complex decision variables for information beamforming $\left(\boldsymbol{w}_{s, d_{\ell}}\right.$ of dimension $N_{t}$ plus the complex variable $q_{s, d_{\ell}}$ ), while the latter involves $2 N_{t}$ complex decision variables $\left(\boldsymbol{w}_{j, d_{\ell}}, j=1,2\right.$, each of dimension $\left.N_{t}\right)$.

For the information transfer during the remaining $1 / t_{2}$ fractional time, the signal (4) received at the $\mathrm{IU}\left(i, d_{j}\right)$ is now specified as

$$
y_{i, d_{j}}=\sum_{\left(s, d_{\ell}\right) \in \mathcal{S}_{I}} \mathbf{h}_{s, i, d_{j}} \boldsymbol{w}_{s, d_{\ell}} s_{s, d_{\ell}}+n_{i, d_{j}} .
$$

By writing down its augmented form:

$$
\left[\begin{array}{l}
y_{i, d_{j}} \\
y_{i, d_{j}}^{*}
\end{array}\right]=\sum_{\left(s, d_{\ell}\right) \in \mathcal{S}_{I}} \mathcal{L}_{s, i, d_{j}}\left(\boldsymbol{w}_{s, d_{\ell}}\right)\left[\begin{array}{l}
s_{s, d_{\ell}} \\
s_{s, d_{\ell}}^{*}
\end{array}\right]+\left[\begin{array}{l}
n_{i, d_{j}} \\
n_{i, d_{j}}^{*}
\end{array}\right],
$$

for

$$
\mathcal{L}_{s, i, d_{j}}\left(\boldsymbol{w}_{s, d_{\ell}}\right) \triangleq\left[\begin{array}{cc}
\mathbf{h}_{s, i, d_{j}} \boldsymbol{w}_{s, d_{\ell}} & 0 \\
0 & \mathbf{h}_{s, i, d_{j}}^{*} \boldsymbol{w}_{s, d_{\ell}}^{*}
\end{array}\right] \in \mathbb{C}^{2 \times 2},
$$

which is a linear operator from $\mathbb{C}^{N_{t} \times 1}$ to $\mathbb{C}^{2 \times 2}$, we can readily determine the augmented covariance of the signal of interest in (29) as

$$
\Phi_{i, d_{j}}\left(\boldsymbol{w}_{i, d_{j}}, q_{i, d_{j}}\right)=\mathcal{L}_{i, i, d_{j}}\left(\boldsymbol{w}_{i, d_{j}}\right) \mathbf{P}_{i, d_{j}} \mathcal{L}_{i, i, d_{j}}^{H}\left(\boldsymbol{w}_{i, d_{j}}\right)
$$

and the augmented covariance of the interference-plus-noise in (29) as

$$
\begin{aligned}
& \Gamma_{i, d_{j}}\left(\boldsymbol{w}_{\mathcal{S}_{I}}, q_{\mathcal{S}_{I}}\right)= \\
& \sum_{\left(s, d_{\ell}\right) \in \mathcal{S}_{I} \backslash\left(i, d_{j}\right)} \mathcal{L}_{s, i, d_{j}}\left(\boldsymbol{w}_{s, d_{\ell}}\right) \mathbf{P}_{s, d_{\ell}} \mathcal{L}_{s, i, d_{j}}^{H}\left(\boldsymbol{w}_{s, d_{\ell}}\right)+\sigma^{2} \mathbf{I}_{2} .
\end{aligned}
$$

The information throughput at user $\left(i, d_{j}\right)$ is then expressed as [12]

$$
\frac{1}{2 t_{2}} r_{i, d_{j}}\left(\boldsymbol{w}_{\mathcal{S}_{I}}, q s\right)
$$


where

$$
\begin{array}{r}
r_{i, d_{j}}\left(\boldsymbol{w}_{\mathcal{S}_{I}}, q s\right)= \\
\ln \left|\mathbf{I}_{2}+\Phi_{i, d_{j}}\left(\boldsymbol{w}_{i, d_{j}}, q_{i, d_{j}}\right)\left(\Gamma_{i, d_{j}}\left(\boldsymbol{w}_{\mathcal{S}_{I}}, q_{\mathcal{S}_{I}}\right)\right)^{-1}\right| .
\end{array}
$$

With $\mathbf{x}_{s, d_{\ell}}^{\mathrm{I}}$ defined by (24), the problem of max-min information throughput optimization subject to the EUs' harvested energy and power constraints is formulated as follows instead of (15):

$$
\begin{array}{r}
\max _{\mathbf{t}=\left(t_{1}, t_{2}\right) \in \mathbb{R}_{+}^{2}, \mathbf{v}_{\mathcal{S}_{E}}, \boldsymbol{w}_{\mathcal{S}_{I}}, q_{\mathcal{S}_{I}}, \gamma} \gamma \quad \text { s.t. } \quad(15 c),(15 d),(26), \\
r_{i, d_{j}}\left(\boldsymbol{w}_{\mathcal{S}_{I}}, q s\right) \geq 2 \gamma t_{2},\left(i, d_{j}\right) \in \mathcal{S}_{I}, \\
\frac{\sum_{j=1}^{K}\left\|\mathbf{v}_{i, e_{j}}\right\|^{2}}{t_{1}}+\frac{\sum_{j=1}^{M}\left\|\boldsymbol{w}_{i, d_{j}}\right\|^{2}}{t_{2}} \leq P, \\
i=1, \ldots, N, \\
\left\|\mathbf{v}_{i, e_{j}}\right\|^{2} \leq P_{\max },\left(i, e_{j}\right) \in \mathcal{S}_{E}, \\
\left\|\boldsymbol{w}_{i, d_{j}}\right\|^{2} \leq P_{\max },\left(i, d_{j}\right) \in \mathcal{S}_{I},
\end{array}
$$

where $(35 b)$ is the counterpart of $(15 b)$ for maximizing the IUs' minimal throughput, while (35c) and (35d)-(35e) correspond to the power constraints (15e) and (15f), respectively. In (35), the constraint (26) is obviously convex, and the constraints (15c), (35c) and (35d)-(35e) are also convex just like their counterparts in (15).

The nonconvex constraint (35b) involves much fewer decision variables than its counterpart (15b) but the former also contains many crossed terms between beamformers and pseudo-covariances that require a different approximation technique.

Let $\left(\mathbf{t}^{(\kappa)}, \gamma^{(\kappa)}, \mathbf{w}_{\mathcal{S}_{I}}^{(\kappa)}, \mathbf{v}_{\mathcal{S}_{E}}^{(\kappa)}, q_{\mathcal{S}_{I}}^{(\kappa)}\right)$ be the feasible point for (35) found from the $(\kappa-1)$ th iteration. The nonconvex constraint (15d) in (35a) is innerly approximated by the convex constraint (20). However, we still have to develop an inner convex approximation of the nonconvex constraint (35b).

\section{A. Path-following iteration}

Use the equivalent representation

$$
r_{i, d_{j}}\left(\boldsymbol{w}_{\mathcal{S}_{I}}, q_{\mathcal{S}_{I}}\right)=f_{i, d_{j}}\left(\boldsymbol{w}_{i, d_{j}}, \mathbf{P}_{i, d_{j}}\right)+g_{i, d_{j}}\left(\boldsymbol{w}_{\mathcal{S}_{I}}, q_{\mathcal{S}_{I}}\right)
$$

in conjunction with

$$
\begin{aligned}
f_{i, d_{j}}\left(\boldsymbol{w}_{i, d_{j}}, \mathbf{P}_{i, d_{j}}\right) & \triangleq \quad \ln \left|\Phi_{i, d_{j}}\left(\boldsymbol{w}_{i, d_{j}}, q_{i, d_{j}}\right)\right| \\
& =2 \ln \left|\mathbf{h}_{i, i, d_{j}} \boldsymbol{w}_{i, d_{j}}\right|^{2}+\ln \left|\mathbf{P}_{i, d_{j}}\right|
\end{aligned}
$$

and

$$
\begin{aligned}
g_{i, d_{j}}\left(\boldsymbol{w}_{\mathcal{S}_{I}}, q_{\mathcal{S}_{I}}\right) \triangleq & \ln \mid\left(\Phi_{i, d_{j}}\left(\boldsymbol{w}_{i, d_{j}}, q_{i, d_{j}}\right)\right)^{-1} \\
& +\left(\Gamma_{i, d_{j}}\left(\boldsymbol{w}_{\mathcal{S}_{I}}, q_{\mathcal{S}_{I}}\right)\right)^{-1} \mid .
\end{aligned}
$$

Using the inequality (64) yields

$$
\begin{aligned}
f_{i, d_{j}}\left(\boldsymbol{w}_{i, d_{j}}, \mathbf{P}_{i, d_{j}}\right) & \geq \\
f_{i, d_{j}}\left(\mathbf{w}_{i, d_{j}}^{(\kappa)}, \mathbf{P}_{i, d_{j}}^{(\kappa)}\right)+4-\frac{2\left|\mathbf{h}_{i, i, d_{j}} \mathbf{w}_{i, d_{j}}^{(\kappa)}\right|^{2}}{\left|\mathbf{h}_{i, i, d_{j}} \boldsymbol{w}_{i, d_{j}}\right|^{2}}-\left\langle\mathbf{P}_{i, d_{j}}^{(\kappa)}, \mathbf{P}_{i, d_{j}}^{-1}\right\rangle & \geq \\
f_{i, d_{j}}\left(\mathbf{w}_{i, d_{j}}^{(\kappa)}, \mathbf{P}_{i, d_{j}}^{(\kappa)}\right)+4-\left\langle\mathbf{P}_{i, d_{j}}^{(\kappa)}, \mathbf{P}_{i, d_{j}}^{-1}\right\rangle & \\
-\frac{2\left|\mathbf{h}_{i, i, d_{j}} \mathbf{w}_{i, d_{j}}^{(\kappa)}\right|^{2}}{2 \Re\left\{\left(\mathbf{w}_{i, d_{j}}^{(\kappa)}\right)^{H} \mathbf{h}_{i, i, d_{j}} \mathbf{h}_{i, i, d_{j}} \boldsymbol{w}_{i, d_{j}}\right\}-\left|\mathbf{h}_{i, i, d_{j}} \mathbf{w}_{i, d_{j}}^{(\kappa)}\right|^{2}} & \triangleq \\
f_{i, d_{j}}^{(\kappa)}\left(\boldsymbol{w}_{i, d_{j}}, \mathbf{P}_{i, d_{j}}\right) & \text { (39) }
\end{aligned}
$$

over the trust region

$$
2 \Re\left\{\left(\mathbf{w}_{i, d_{j}}^{(\kappa)}\right)^{H} \mathbf{h}_{i, i, d_{j}}^{H} \mathbf{h}_{i, i, d_{j}} \boldsymbol{w}_{i, d_{j}}\right\}-\left|\mathbf{h}_{i, i, d_{j}} \mathbf{w}_{i, d_{j}}^{(\kappa)}\right|^{2}>0 .
$$

Furthermore, using the inequality (62) in the Appendix yields

$$
\begin{aligned}
g_{i, d_{j}}\left(\boldsymbol{w}_{\mathcal{S}_{I}}, q_{\mathcal{S}_{I}}\right) & \geq \\
g_{i, d_{j}}\left(\mathbf{w}_{\mathcal{S}_{I}}^{(\kappa)}, q_{\mathcal{S}_{I}}^{(\kappa)}\right)+2-\left\langle\mathbf{B}_{i, d_{j}}^{(\kappa)}, \Phi_{i, d_{j}}\left(\boldsymbol{w}_{i, d_{j}}, q_{i, d_{j}}\right)\right\rangle & \\
-\left\langle\mathbf{C}_{i, d_{j}}^{(\kappa)}, \Gamma_{i, d_{j}}\left(\boldsymbol{w}_{\mathcal{S}_{I}}, q_{\mathcal{S}_{I}}\right)\right\rangle & = \\
g_{i, d_{j}}\left(\mathbf{w}_{\mathcal{S}_{I}}^{(\kappa)}, q_{\mathcal{S}_{I}}^{(\kappa)}\right)+2-\sigma^{2}\left\langle\mathbf{C}_{i, d_{j}}^{(\kappa)}\right\rangle & \\
-\sum_{\left(s, d_{\ell}\right) \in \mathcal{S}_{I}}\left\langle\left[\chi_{i, d_{j}, s, d_{\ell}}\left(\boldsymbol{w}_{s, d_{\ell}}\right)\right]^{2}, \mathbf{P}_{s, d_{\ell}}\right\rangle &
\end{aligned}
$$

where

$$
\begin{aligned}
0 \prec \mathbf{B}_{i, d_{j}}^{(\kappa)} \triangleq & \left(\Phi_{i, d_{j}}\left(\mathbf{w}_{i, d_{j}}^{(\kappa)}, q_{i, d_{j}}^{(\kappa)}\right)\right)^{-1} \\
& -\left(\Phi_{i, d_{j}}\left(\mathbf{w}_{i, d_{j}}^{(\kappa)}, q_{i, d_{j}}^{(\kappa)}\right)+\Gamma_{i, d_{j}}\left(\mathbf{w}_{\mathcal{S}_{I}}^{(\kappa)}, q_{\mathcal{S}_{I}}^{(\kappa)}\right)\right)^{-1} \\
0 \prec \mathbf{C}_{i, d_{j}}^{(\kappa)} \triangleq & \left(\Gamma_{i, d_{j}}\left(\mathbf{w}_{\mathcal{S}_{I}}^{(\kappa)}, q_{\mathcal{S}_{I}}^{(\kappa)}\right)\right)^{-1} \\
& -\left(\Phi_{i, d_{j}}\left(\mathbf{w}_{i, d_{j}}^{(\kappa)}, q_{i, d_{j}}^{(\kappa)}\right)+\Gamma_{i, d_{j}}\left(\mathbf{w}_{\mathcal{S}_{I}}^{(\kappa)}, q_{\mathcal{S}_{I}}^{(\kappa)}\right)\right)^{-1}
\end{aligned}
$$

and

$$
\begin{array}{r}
\chi_{i, d_{j}, i, d_{j}}\left(\boldsymbol{w}_{i, d_{j}}\right) \triangleq \mathcal{L}_{i, i, d_{j}}^{*}\left(\boldsymbol{w}_{i, d_{j}}\right)\left(\mathbf{B}_{i, d_{j}}^{(\kappa)}\right)^{1 / 2}, \\
\chi_{i, d_{j}, s, d_{\ell}}\left(\boldsymbol{w}_{s, d_{\ell}}\right)=\mathcal{L}_{s, i, d_{j}}^{*}\left(\boldsymbol{w}_{s, d_{\ell}}\right)\left(\mathbf{C}_{i, d_{j}}^{(\kappa)}\right)^{1 / 2}, \\
\left(s, d_{\ell}\right) \in \mathcal{S}_{I} \backslash\left(i, d_{j}\right) .
\end{array}
$$

Let us introduce the positive definite matrix variables $\mathbf{X}_{i, d_{j}, s, d_{\ell}}$ of size $2 \times 2$ satisfying the semi-definite constraints of

$$
\begin{gathered}
{\left[\chi_{i, d_{j}, s, d_{\ell}}\left(\boldsymbol{w}_{s, d_{\ell}}\right)\right]^{2} \preceq \mathbf{X}_{i, d_{j}, s, d_{\ell}},\left(s, d_{\ell}\right) \in \mathcal{S}_{I}} \\
\left.\Leftrightarrow \quad \begin{array}{cc}
\mathbf{X}_{i, d_{j}, s, d_{\ell}} & \chi_{i, d_{j}, s, d_{\ell}}\left(\boldsymbol{w}_{s, d_{\ell}}\right) \\
\left(\chi_{i, d_{j}, s, d_{\ell}}\left(\boldsymbol{w}_{s, d_{\ell}}\right)\right)^{H} & \mathbf{I}_{2}
\end{array}\right] \succeq 0, \\
\left(s, d_{\ell}\right) \in \mathcal{S}_{I} .
\end{gathered}
$$

Then, by using the inequality (65), we arrive at:

$$
\begin{array}{r}
\left\langle\left[\chi_{i, d_{j}, s, d_{\ell}}\left(\boldsymbol{w}_{s, d_{\ell}}\right)\right]^{2}, \mathbf{P}_{s, d_{\ell}}\right\rangle \leq \\
\left\langle\mathbf{X}_{i, d_{j}, s, d_{\ell}}, \mathbf{P}_{s, d_{\ell}}\right\rangle \leq \\
\frac{1}{4} \|\left(\mathbf{X}_{i, d_{j}, s, d_{\ell}}^{(\kappa)}\right)^{-1 / 2}\left(\mathbf{X}_{i, d_{j}, s, d_{\ell}} \mathbf{P}_{s, d_{\ell}}^{(\kappa)}\right. \\
\left.+\mathbf{X}_{i, d_{j}, s, d_{\ell}}^{(\kappa)} \mathbf{P}_{s, d_{\ell}}\right)\left(\mathbf{P}_{s, d_{\ell}}^{(\kappa)}\right)^{-1 / 2} \|^{2}, \\
\left(s, d_{\ell}\right) \in \mathcal{S}_{I},
\end{array}
$$


for

$$
\mathbf{X}_{i, d_{j}, s, d_{\ell}}^{(\kappa)}=\left[\chi_{i, d_{j}, s, d_{\ell}}\left(\mathbf{w}_{s, d_{\ell}}^{(\kappa)}\right)\right]^{2},\left(s, d_{\ell}\right) \in \mathcal{S}_{I} .
$$

Hence,

$$
g_{i, d_{j}}\left(\boldsymbol{w}_{\mathcal{S}_{I}}, q_{\mathcal{S}_{I}}\right) \geq g_{i, d_{j}}^{(\kappa)}\left(\mathbf{X}_{\mathcal{S}_{I}}, q_{\mathcal{S}_{I}}\right)
$$

for

$$
\begin{array}{r}
g_{i, d_{j}}^{(\kappa)}\left(\mathbf{X}_{\mathcal{S}_{I}}, q_{\mathcal{S}_{I}}\right) \triangleq \\
\frac{1}{4} \sum_{\left(s, d_{\ell}\right) \in \mathcal{S}_{I}} \|\left(\mathbf{X}_{i, d_{j}, s, d_{\ell}}^{(\kappa)}\right)^{-1 / 2}\left(\mathbf{X}_{i, d_{j}, s, d_{\ell}} \mathbf{P}_{s, d_{\ell}}^{(\kappa)}\right. \\
\left.+\mathbf{X}_{i, d_{j}, s, d_{\ell}}^{(\kappa)} \mathbf{P}_{s, d_{\ell}}\right)\left(\mathbf{P}_{\mathcal{S}_{I}, d_{\ell}}^{(\kappa)}, q_{\mathcal{S}_{I}}^{(\kappa)}\right)+2- \\
{ }^{-1 / 2} \|_{F}^{2} .
\end{array}
$$

At the $\kappa$ th iteration we solve the following convex optimization problem to generate the next iterative point $\left(\mathbf{t}^{(\kappa+1)}, \gamma^{(\kappa+1)}, \mathbf{w}_{\mathcal{S}_{I}}^{(\kappa+1)}, \mathbf{v}_{\mathcal{S}_{E}}^{(\kappa+1)}, q_{\mathcal{S}}^{(\kappa+1)}\right)$

$$
\begin{aligned}
& \max _{\mathbf{t}, \boldsymbol{w}_{\mathcal{S}_{I}}, q_{\mathcal{S}_{I}}, \mathbf{v}_{\mathcal{S}_{E}}, \gamma, \mathbf{X}_{\mathcal{S}_{I}}} \gamma \quad \text { s.t. } \quad(15 d),(20),(26),(35 c), \\
& (35 d)-(35 e),(40),(45), \\
& f_{i, d_{j}}^{(\kappa)}\left(\boldsymbol{w}_{i, d_{j}}, \mathbf{P}_{i, d_{j}}\right)+g_{i, d_{j}}^{(\kappa)}\left(\mathbf{X}_{\mathcal{S}_{I}}, q_{\mathcal{S}_{I}}\right) \geq \\
& \frac{\gamma^{(\kappa)} t_{2}^{(\kappa)}}{2}\left(\frac{\gamma}{\gamma^{(\kappa)}}+\frac{t_{2}}{t_{2}^{(\kappa)}}\right)^{2},\left(i, d_{j}\right) \in \mathcal{S}_{I},
\end{aligned}
$$

The computational complexity of this convex problem is (22) is determined by $n_{v}=3+N[M(N+t+1+3 M)+K]$ and $m_{c}=1+N(2 K+4 M+1)$.

Note by observing (39) and (49) that the LHS of (51b) is a concave lower bounding approximation of the LHS of (35b), while by (18), the RHS of (51b) is a convex upper-bounding approximation of the RHS of (35b). Hence in fact the convex constraint (51b) is an inner approximation of the nonconvex constraint (35b). The convex problem (51) is then seen as an inner approximation of the nonconvex problem (35). Then $\gamma^{(\kappa+1)}>\gamma^{(\kappa)}$ as far as $\gamma^{(\kappa+1)} \neq \gamma^{(\kappa)}$ because $\quad\left(\mathbf{t}^{(\kappa+1)}, \gamma^{(\kappa+1)}, \mathbf{w}_{\mathcal{S}_{I}}^{(\kappa+1)}, \mathbf{v}_{\mathcal{S}_{E}}^{(\kappa+1)}, q_{\mathcal{S}}^{(\kappa+1)}\right) \quad$ and $\left(\mathbf{t}^{(\kappa)}, \gamma^{(\kappa)}, \mathbf{w}_{\mathcal{S}_{I}}^{(\kappa)}, \mathbf{v}_{\mathcal{S}_{E}}^{(\kappa)}, q_{\mathcal{S}_{I}}^{(\kappa)}\right)$ are the optimal solution and a feasible point for (51). As such, the sequence $\left\{\left(\mathbf{t}^{(\kappa)}, \gamma^{(\kappa)}, \mathbf{w}_{\mathcal{S}_{I}}^{(\kappa)}, \mathbf{v}_{\mathcal{S}_{E}}^{(\kappa)}, q_{\mathcal{S}_{I}}^{(\kappa)}\right)\right\}$ generated by $(51)$ is of improved feasible points for the nonconvex problem (35) and it converges at least to a point satisfying the KKT condition of optimality [38].

\section{B. Alternating descent iteration}

One can see that the function $r_{i, d_{j}}\left(\boldsymbol{w}_{\mathcal{S}_{I}}, q_{\mathcal{S}_{I}}\right)$ defined by (34) is complex. We therefore develop an alternating procedure for its more efficient computation.

1) Alternating optimization in $\boldsymbol{w}_{\mathcal{S}}$ : By fixing $q_{\mathcal{S}_{I}}=q_{\mathcal{S}_{I}}^{(\kappa)}$, we address the problem

$$
\begin{array}{rrr}
\max _{\mathbf{t}, \boldsymbol{w}_{\mathcal{S}_{I}}, \mathbf{v}_{\mathcal{S}_{E}}, \gamma} \gamma & \text { s.t. } & (15 c),(15 d),(35 c),(35 d)-(35 e),(52 \mathrm{a}) \\
& r_{i, d_{j}}\left(\boldsymbol{w}_{\mathcal{S}_{I}}, q_{\mathcal{S}_{I}}^{(\kappa)}\right) \geq 2 \gamma t_{2},\left(i, d_{j}\right) \in \mathcal{S}_{I} .(52 \mathrm{~b})
\end{array}
$$

Using the inequality (60) in the Appendix yields

$$
\begin{aligned}
r_{i, d_{j}}\left(\boldsymbol{w}_{\mathcal{S}_{I}}, q_{\mathcal{S}_{I}}^{(\kappa)}\right) & \geq \\
r_{i, d_{j}}\left(\mathbf{w}_{\mathcal{S}_{I}}^{(\kappa)}, q_{\mathcal{S}_{I}}^{(\kappa)}\right)-\left\langle\Phi_{i, d_{j}}\left(\mathbf{w}_{i, d_{j}}^{(\kappa)}, q_{i, d_{j}}^{(\kappa)}\right),\right. & \left.\left(\Gamma_{i, d_{j}}\left(\mathbf{w}_{\mathcal{S}_{I}}^{(\kappa)}, q_{\mathcal{S}_{I}}^{(\kappa)}\right)\right)^{-1}\right\rangle \\
+2 \Re\left\{\left\langle\mathcal{L}_{i, i, d_{j}}\left(\boldsymbol{w}_{i, d_{j}}\right) \mathbf{P}_{i, d_{j}}^{(\kappa)} \mathcal{L}_{i, i, d_{j}}^{H}\left(\mathbf{w}_{i, d_{j}}^{(\kappa)}\right)\right.\right. & \left.\left.\times\left(\Gamma_{i, d_{j}}\left(\mathbf{w}_{\mathcal{S}_{I}}^{(\kappa)}, q_{\mathcal{S}_{I}}^{(\kappa)}\right)\right)^{-1}\right\rangle\right\} \\
-\left\langle\left(\Gamma_{i, d_{j}}\left(\mathbf{w}_{\mathcal{S}_{I}}^{(\kappa)}, q_{\mathcal{S}_{I}}^{(\kappa)}\right)\right)^{-1}-\left(\Phi_{i, d_{j}}\left(\mathbf{w}_{i, d_{j}}^{(\kappa)}, q_{i, d_{j}}^{(\kappa)}\right)\right.\right. & \\
\left.+\Gamma_{i, d_{j}}\left(\mathbf{w}_{\mathcal{S}_{I}}^{(\kappa)}, q_{\mathcal{S}_{I}}^{(\kappa)}\right)\right)^{-1}, & \\
\left.\Phi_{i, d_{j}}\left(\boldsymbol{w}_{i, d_{j}}, q_{i, d_{j}}^{(\kappa)}\right)+\Gamma_{i, d_{j}}\left(\boldsymbol{w}_{\mathcal{S}_{I}}, q_{\mathcal{S}_{I}}^{(\kappa)}\right)\right\rangle & \triangleq \\
\tilde{r}_{i, d_{j}}^{(\kappa)}\left(\boldsymbol{w}_{\mathcal{S}_{I}}\right) . &
\end{aligned}
$$

We solve the following convex problem for generating the next feasible point $\left(\mathbf{t}^{(\kappa+1)}, \bar{\gamma}^{(\kappa+1)}, \mathbf{w}_{\mathcal{S}_{I}}^{(\kappa+1)}, \mathbf{v}_{\mathcal{S}_{E}}^{(\kappa+1)}\right)$ for (35):

$$
\begin{gathered}
\max _{\mathbf{t}, \boldsymbol{w}_{\mathcal{S}_{I}, \mathbf{v} \mathcal{S}_{E}, \gamma} \gamma} \text { s.t. } \quad(15 c),(20),(35 c),(35 d)-(35 e),(54 \mathrm{a}) \\
\tilde{r}_{i, d_{j}}^{(\kappa)}\left(\boldsymbol{w}_{\mathcal{S}_{I}}\right) \geq \frac{\gamma^{(\kappa)} t_{2}^{(\kappa)}}{2}\left(\frac{\gamma}{\gamma^{(\kappa)}}+\frac{t_{2}}{t_{2}^{(\kappa)}}\right)^{2},\left(i, d_{j}\right) \in \mathcal{S}_{I} .(54 \mathrm{~b})
\end{gathered}
$$

The computational complexity of this convex problem is (22) determined by $n_{v}=3+N N_{t}(M+K)$ and $m_{c}=N(3 K+$ $2 M+1)$.

Since the convex constraint (54b) is an inner approximation of the nonconvex constraint (52b), the convex problem (54) is seen as an inner approximation of the nonconvex problem (52). We then have

$$
\bar{\gamma}^{(\kappa+1)} \geq \gamma^{(\kappa)},
$$

because they are the optimal and a feasible value for (54).

2) Alternating optimization in $q_{\mathcal{S}}$ : By fixing $\left(\mathbf{t}, \boldsymbol{w}_{\mathcal{S}_{I}}, \mathbf{v}_{\mathcal{S}_{E}}\right)=\left(\mathbf{t}^{(\kappa+1)}, \mathbf{w}_{\mathcal{S}_{I}}^{(\kappa+1)}, \mathbf{v}_{\mathcal{S}_{E}}^{(\kappa+1)}\right)$, we address the problem

$$
\begin{array}{r}
\max _{q_{\mathcal{S}_{I}}, \gamma} \gamma \quad \text { s.t. } \quad(26), \\
r_{i, d_{j}}\left(\mathbf{w}_{\mathcal{S}_{I}}^{(\kappa+1)}, q_{\mathcal{S}_{I}}\right) \geq 2 \gamma t_{2}^{(\kappa+1)},\left(i, d_{j}\right) \in \mathcal{S}_{I} .
\end{array}
$$

Using the inequality (61) in the Appendix yields

$$
\begin{aligned}
r_{i, d_{j}}\left(\mathbf{w}_{\mathcal{S}_{I}}^{(\kappa+1)}, q_{\mathcal{S}_{I}}\right) & \geq \\
r_{i, d_{j}}\left(\mathbf{w}_{\mathcal{S}_{I}}^{(\kappa+1)}, q_{\mathcal{S}_{I}}^{(\kappa)}\right)+4 & \\
-\left\langle\left(\Gamma_{i, d_{j}}\left(\mathbf{w}_{\mathcal{S}_{I}}^{(\kappa+1)}, q_{\mathcal{S}_{I}}^{(\kappa)}\right)\right)^{-1}, \Gamma_{i, d_{j}}\left(\mathbf{w}_{\mathcal{S}_{I}}^{(\kappa+1)}, q_{\mathcal{S}_{I}}\right)\right\rangle & \\
-\left\langle\Phi_{i, d_{j}}\left(\mathbf{w}_{i, d_{j}}^{(\kappa+1)}, q_{i, d_{j}}^{(\kappa)}\right)+\Gamma_{i, d_{j}}\left(\mathbf{w}_{\mathcal{S}_{I}}^{(\kappa+1)}, q_{\mathcal{S}_{I}}^{(\kappa)}\right),\right. & \\
\left.\left(\Phi_{i, d_{j}}\left(\mathbf{w}_{i, d_{j}}^{(\kappa+1)}, q_{i, d_{j}}\right)+\Gamma_{i, d_{j}}\left(\mathbf{w}_{\mathcal{S}_{I}}^{(\kappa+1)}, q_{\mathcal{S}_{I}}\right)\right)^{-1}\right\rangle & \triangleq \\
\hat{r}_{i, d_{j}}^{(\kappa)}\left(q_{\mathcal{S}_{I}}\right) . &
\end{aligned}
$$

We then solve the following convex optimization problem for generating the next feasible point $\left(q_{\mathcal{S}}^{(\kappa+1)}, \gamma^{(\kappa+1)}\right)$ for (35):

$$
\begin{array}{r}
\max _{q_{\mathcal{S}_{I}, \gamma}, \gamma} \gamma \text { s.t. } \quad(26), \\
\hat{r}_{i, d_{j}}^{(\kappa)}\left(q_{\mathcal{S}_{I}}\right) \geq 2 \gamma t_{2}^{(\kappa+1)},\left(i, d_{j}\right) \in \mathcal{S}_{I} .
\end{array}
$$


The computational complexity of this convex problem is (22) determined by $n_{v}=1+N M$ and $m_{c}=3 N M$.

Note that we have $\gamma^{(\kappa+1)}>\bar{\gamma}^{(\kappa+1)} \geq \gamma^{(\kappa)}$ provided that $\gamma^{(\kappa+1)}>\bar{\gamma}^{(\kappa+1)}$, hence the sequence $\left\{\left(\mathbf{t}^{(\kappa)}, \gamma^{(\kappa)}, \mathbf{w}_{\mathcal{S}_{I}}^{(\kappa)}, \mathbf{v}_{\mathcal{S}_{E}}^{(\kappa)}, q_{\mathcal{S}_{I}}^{(\kappa)}\right)\right\}$ is of feasible points for the nonconvex problem (35), which converges to a feasible point satisfying the KKT conditions for one of two variable sets $\left(\mathbf{t}, \mathbf{v}_{\mathcal{S}_{E}}, \boldsymbol{w}_{\mathcal{S}_{I}}\right)$ and $q_{\mathcal{S}_{I}}$, when the other is held fixed.

\section{Generating a good feasible point for (35)}

It is important to generate a good feasible point for (35). For this we fix $\mathbf{t}^{(0)}$ to satisfy $(15 \mathrm{~d})$ and $q_{\mathcal{S}_{I}}^{(0)}$ (for instance $\left.q_{s, d_{\ell}}^{(0)} \equiv 0.2\right)$ and reasonable $\gamma^{(0)}$. We then randomly generate $w_{\mathcal{S}_{I}}^{(0)}$ and $v_{\mathcal{S}_{E}}^{(0)}$ satisfying the convex constraints $(35 \mathrm{c})-(35 \mathrm{e})$. Let us set

$$
\mathbf{P}_{i, d_{j}}^{(\kappa)} \equiv\left[\begin{array}{cc}
1 & q_{i, d_{j}}^{(0)} \\
\left(q_{i, d_{j}}^{(0)}\right)^{*} & 1
\end{array}\right]
$$

We then iterate

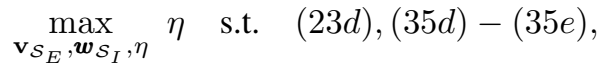

$$
\begin{aligned}
& \tilde{r}_{i, d_{j}}^{(\kappa)}\left(\boldsymbol{w}_{\mathcal{S}_{I}}\right) \geq 2 \gamma_{0} t_{2}^{(0)} \eta,\left(i, d_{j}\right) \in \mathcal{S}_{I}, \\
& \frac{\sum_{j=1}^{K}\left\|\mathbf{v}_{i, e_{j}}\right\|^{2}}{t_{1}^{(0)}}+\frac{\sum_{j=1}^{M}\left\|\boldsymbol{w}_{i, d_{j}}\right\|^{2}}{t_{2}^{(0)}} \leq P, i=1, \ldots, N \text {, }
\end{aligned}
$$

until we have $\eta \geq 1$ for guaranteeing that $\left(\mathbf{t}^{(0)}, \gamma^{(0)} \eta, w_{\mathcal{S}_{I}}^{(\kappa)}, v_{\mathcal{S}_{E}}^{(\kappa)}, q_{\mathcal{S}_{I}}^{(\kappa)}\right)$ is feasible for (35).

\section{Algorithm}

For optimizing a trade-off between the convergence speed and the solution optimality we propose Algorithm 2, which uses the alternating optimization until its convergence and then switches to the path-following optimization in order to converge at least to a locally optimal solution.

\footnotetext{
Algorithm 2 s-IGS algorithm for (35)

1: Initialization: Fix $q_{\mathcal{S}_{I}}^{(0)}$. Set $\kappa:=0$ and then iterate (59) for finding a good initial feasible point $\left(\mathbf{v}_{\mathcal{S}_{E}}^{(0)}, \boldsymbol{w}_{\mathcal{S}_{I}}^{(0)}, \mathbf{t}^{(0)}\right)$ for (35)

2: Repeat until convergence of the objective in (35): Solve the convex optimization problems (54) and (58) of alternating optimization to generate the feasible point $\left(\mathbf{v}_{\mathcal{S}_{E}}^{(\kappa+1)}, \boldsymbol{w}_{\mathcal{S}_{I}}^{(\kappa+1)}, \mathbf{t}^{(\kappa+1)}\right)$ for (35); Reset $\kappa:=\kappa+1$.

3: Repeat until convergence of the objective in (35): Solve the convex optimization problem (51) of pathfollowing optimization to generate the feasible point $\left(\mathbf{v}_{\mathcal{S}_{E}}^{(\kappa+1)}, \boldsymbol{w}_{\mathcal{S}_{I}}^{(\kappa+1)}, \mathbf{t}^{(\kappa+1)}\right)$ for (35); Reset $\kappa:=\kappa+1$.

4: Output $\mathbf{t}=t^{(\kappa)}, \mathbf{v}_{\mathcal{S}_{E}}=\mathbf{v}_{\mathcal{S}_{E}}^{(\kappa)}$, and $\boldsymbol{w}_{\mathcal{S}_{I}}=\mathbf{w}_{\mathcal{S}_{I}}^{(\kappa)}$.
}

\section{Performance Results}

In all our simulations we consider networks of three cells $(N=3)$. The channel spanning from a BS to a user at a distance of $d$ meters is expressed as $\sqrt{10^{-\sigma_{\mathrm{PL}} / 10}} \tilde{h}$, where $\sigma_{\mathrm{PL}}=30+10 \beta \log _{10}(d)$ is the path-loss in $\mathrm{dB}$, and $\tilde{h}$ is the Rician fading channel gain associated with a Rician factor of $10 \mathrm{~dB}$ for the EUs served by that BS only. Otherwise, $\tilde{h}$ is the normalized Rayleigh fading channel gain. The path-loss exponent $\beta$ is set to 3 for the Rician channels and to 2 for the Rayleigh channels. The power of the signal received by the UEs must exceed the threshold of $-21 \mathrm{dBm}$ with $13 \mathrm{~nm}$ CMOS technology [4] to facilitate EH. We set $e_{\min }=-20$ $\mathrm{dBm}, \zeta=0.5, P=35 \mathrm{dBm}$. The bandwidth is set to $B=20$ $\mathrm{MHz}$, the carrier frequency is set to $2 \mathrm{GHz}$, and the power spectral density of noise is $-174 \mathrm{dBm} / \mathrm{Hz}$.

\section{A. NOMA favored scenario}

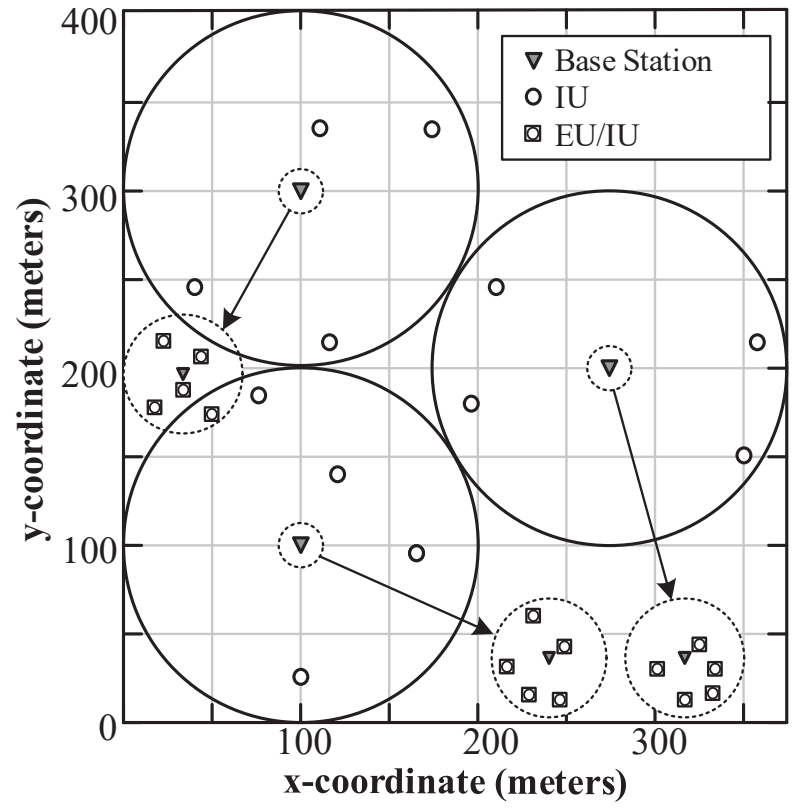

Fig. 2: NOMA favored scenario

Fig. 2 illustrates a scenario, where $K$ EUs $\left(i, e_{j}\right), j=$ $1, \ldots, K$ also act as the first $K$ IUs. The other $K$ IUs $\left(i, d_{K+j}\right), j=1, \ldots, K$ in each cells are distributed near the cell boundary. Those IUs which are located near the cellboundary, are not only in poorer channel conditions than the IUs $\left(i, d_{j}\right), j=1, \ldots, K$ but are then subject to intercellinterference. By bringing about the differentiated channel conditions between the near IUs $\left(i, d_{j}\right), j=1, \ldots, K$ and far IUs $\left(i, d_{K+j}\right), j=1, \ldots, K$, such scenario favours NOMA, helping it to perform better than the conventional OMA. Both NOMA and OMA use proper Gaussian signal for carrying information, i.e. $q_{s, d_{\ell}} \equiv 0$ in (25) so the information signal $\mathbf{x}_{s, d_{\ell}}^{\mathbf{I}}$ defined by (24) or (5) for $\boldsymbol{w}_{2, s, d_{\ell}} \equiv 0$ is generated by linearly beamforming of a normalized proper Gaussian source $s_{s, d_{\ell}}$. Under OMA, each IU $\left(i, d_{j}\right), j=1, \ldots, 2 K$ decodes its own information $s_{i, d_{j}}$ while under NOMA each pair of IUs $\left(i, d_{j}\right)$ and $\left(i, d_{K+j}\right), j=1, \ldots, K$ decode the information 
$s_{i, d_{K+j}}$ for the IU $\left(i, d_{K+j}\right)$ and then the IU $\left(i, d_{j}\right)$ subtracts $s_{i, d_{K+j}}$ from its interference in decoding its own information $s_{i, d_{j}}$.

Fig. 3 characterizes the convergence behaviour of the proposed Algorithms for $N_{t}=6$ and $K=3$, i.e. each BS is equipped with $N_{t}=6 \mathrm{DL}$ TAs and there are a total of 27 users served by the network. The NOMA PGS algorithm [11] converges rapidly as a benefit of the efficient approximation of the logarithmic functions. The convergence rate of the IGS and s-IGS algorithms is similar, but the computational complexity of the latter is significantly lower.

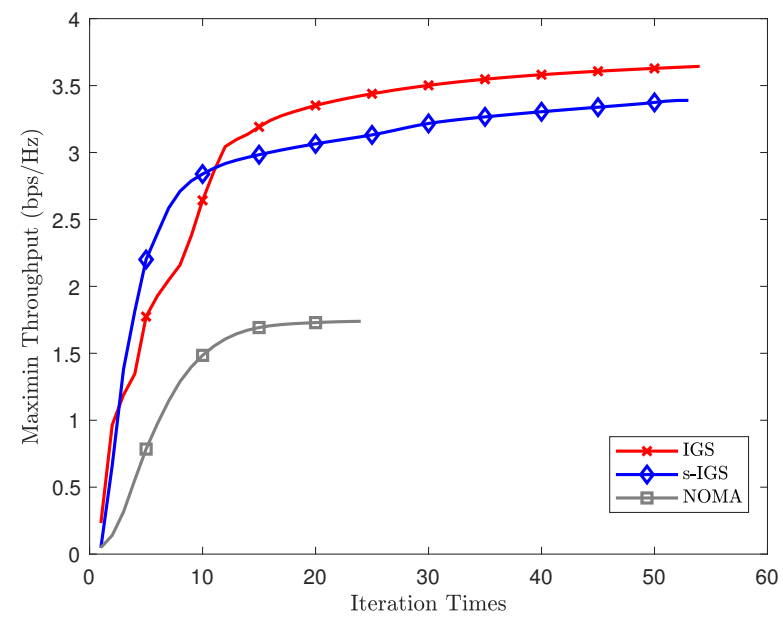

Fig. 3: Convergence for IGS, s-IGS and NOMA algorithms

Fig. 4 plots the achievable minimum throughput under different numbers $N_{t}$ of DL TAs for $K=2$ (18 users in total) and $K=3$ (27 users in total). Both the IGS and sIGS outperform NOMA [11]. IGS outperforms s-IGS since the latter is a particular class of the former. All of them still benefit from the spatial diversity associated with the number $N_{t}$ of BS TAs. This figure also shows the efficiency of the time fraction optimization as IGS, s-IGS and NOMA outperform their counter parts IGS $\left(t_{1}=t_{2}=2\right)$, s-IGS $\left(t_{1}=t_{2}=2\right)$ and NOMA $\left(t_{1}=t_{2}=2\right)$, respectively, which use the half of the time-slot for power transfer and the remaining half for information transfer.

We now examine the achievable minimum throughput upon varying the $\mathrm{BS}$ transmit power budget $P$ in Fig. 5 under $\left(N_{t}, K\right)=(6,3)$. Both the IGS and s-IGA exploit the available transmit power much better than NOMA since the latter cannot use the total affordable power budget because its achievable minimum throughput is not sensitive to $P \geq 33$ $\mathrm{dBm}$. By contrast, by employing additional beamformers $\boldsymbol{w}_{2, s, d_{\ell}}$ for the conjugate proper Gaussian information source $s_{s, d_{\ell}}^{*}$ in (5) or optimizing the pseudo-covariance $q_{s, d_{\ell}}$ in (25), IGS allows the total power budget be exploited for improving its throughput. Naturally, beyond a certain threshold, namely $P=41 \mathrm{dBm}$ in Fig. 5, its performance also becomes saturated. This should not be a surprise for interference-limited networks.

Fig. 6 portrays the users' max-min throughput under $\left(N_{t}, K\right)=(6,3)$ upon varying the $\mathrm{EH}$ threshold $e_{\min }$ to show

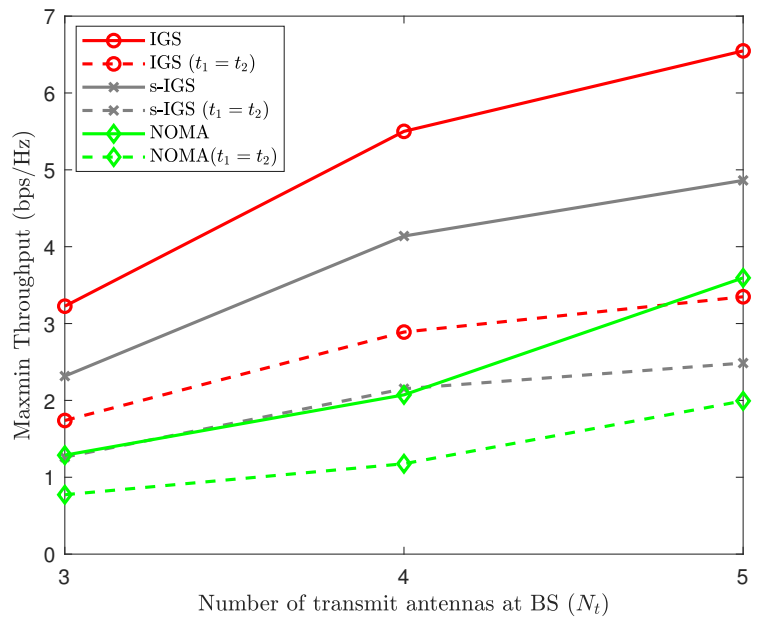

(a)

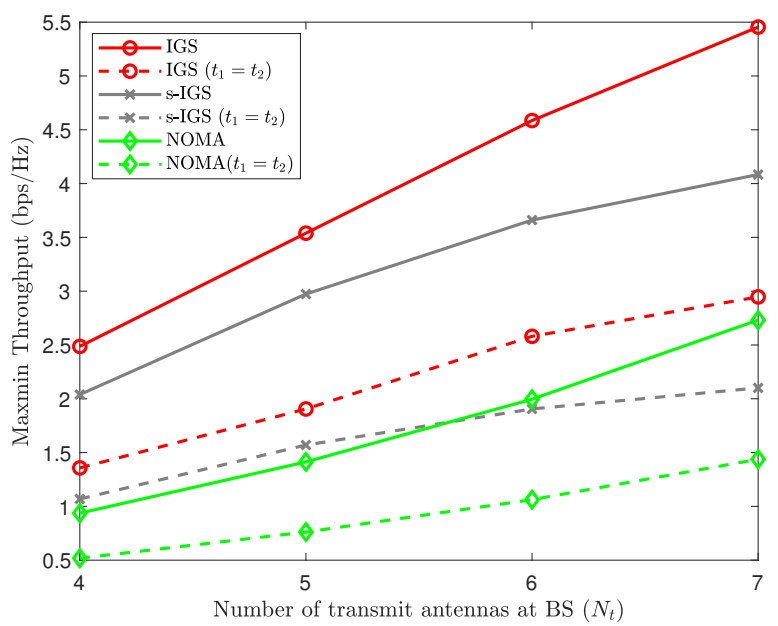

(b)

Fig. 4: Achievable minimum throughput vs number $N_{t}:(a)$ $K=2$ (18 users); (b) $K=3$ (27 users)

the impact of the latter imposed on the former. As expected, the increase of the latter degrades the performance of the former.

Table I provides the rounded average number of iterations required required for the convergence of the three algorithms for $K=3$ under different number of BS TAs $N_{t}$. For lower $N_{t}$ the feasibility set becomes narrower, which forces all algorithms to converge slower.

TABLE I: The rounded average number of iterations for the convergence under NOMA favored scenario

\begin{tabular}{|l|c|c|c|c|}
\hline & $N_{t}=4$ & $N_{t}=5$ & $N_{t}=6$ & $N_{t}=7$ \\
\hline IGS & 30 & 31 & 20 & 20 \\
\hline S-IGS & 25 & 28 & 31 & 18 \\
\hline NOMA & 24 & 22 & 15 & 18 \\
\hline
\end{tabular}




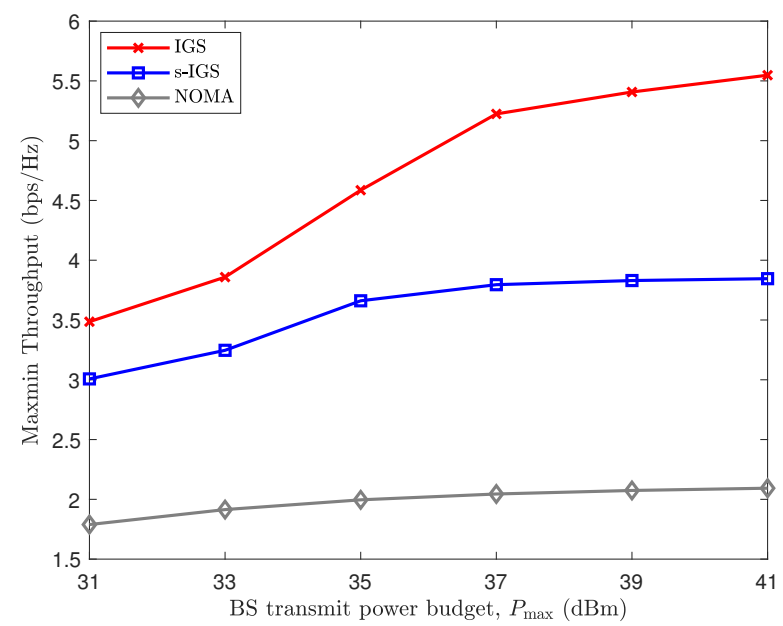

Fig. 5: Achievable minimum throughput vs BS transmit power budget $P$

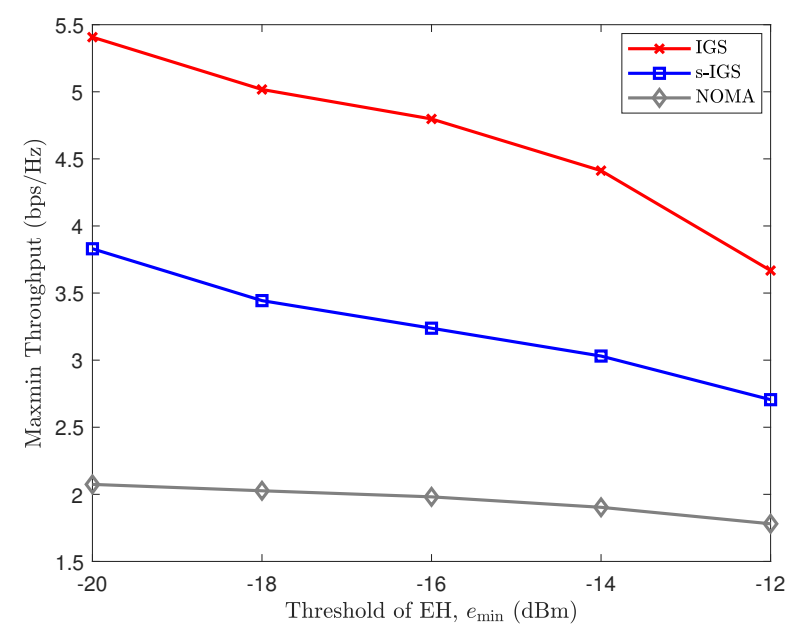

Fig. 6: Achievable minimum throughput vs BS transmit power budget $P$

\section{B. General scenario}

Fig. 7 illustrates a general scenario, where $M$ IUs are located outside the EH zone, hence they cannot act as EUs. The IUs' channel conditions are not differentiated, therefore NOMA is inefficient. We thus compare IGS and s-IGS to the conventional PGS orthogonal multiple access (OMA), in which IU decodes its own message only.

Fig 8 shows the achievable minimum throughput for IGS, s-IGS and PGS OMA for different values of $N_{t}$. There are $K=2$ EUs and $M=4$ IUs for simulating Fig. 8(a), and $K=3$ EUs and $M=6$ IUs for simulating Fig. 8(b). As expected, IGS is the best performer, followed by s-IGS, while PGS OMA is the worst performer. Similarly to Fig. 4, this figure also includes the performance of IGS, s-IGS and PGS OMA at $t_{1}=t_{2}=2$ to show the efficiency of the time fraction optimization.

Fig. 9 provides the achievable minimum throughput for varying values of the $\mathrm{BS}$ transmit power budget $P$. All three

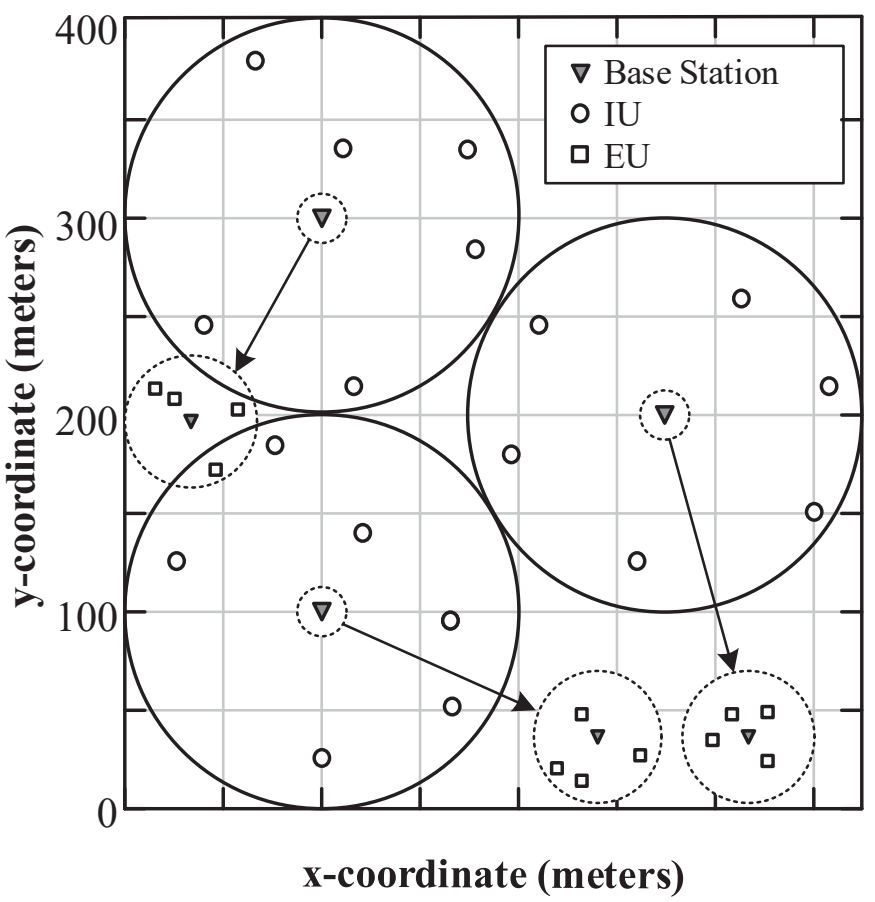

Fig. 7: OMA favored scenario

algorithms are capable of exploiting the affordable power budget to compensate for the increased distance from the BS to the IUs that makes the pathloss higher.

Finally, Table II provides the rounded average number of iterations for the convergence of IGS, s-IGS and PGS OMA for $(K, M)=(3,6)$ and different values of $N_{t}$.

TABLE II: The rounded average number of iterations for the convergence under general scenario

\begin{tabular}{|l|c|c|c|c|}
\hline & $N_{t}=4$ & $N_{t}=5$ & $N_{t}=6$ & $N_{t}=7$ \\
\hline IGS & 17 & 16 & 19 & 18 \\
\hline S-IGS & 16 & 20 & 21 & 19 \\
\hline PGS OMA & 6 & 8 & 10 & 11 \\
\hline
\end{tabular}

\section{CONCLusions}

We have involved improper Gaussian signaling (IGS), in both general format and a particular format (s-IGS), for improving the information throughput of a multi-cell energyharvesting enabled network, which aims for transferring both information and energy over the same wireless channels within a time slot. In contrast to NOMA, which improves the network throughput by allowing the users of better channel condition to access and decode the information of the users of poorer channel condition, IGS is capable of improving the network throughput more substantially than NOMA, maintaining the users' secrecy under OMA. Although the problem of max-min information user throughput subject to the EH thresholds and power budget is much more computationally challenging than its NOMA counterpart, the paper has developed path-following algorithms for its computation, which converge at least to a locally optimal solution. The numerical examples provided for networks serving 18 users and 27 users have confirmed the 


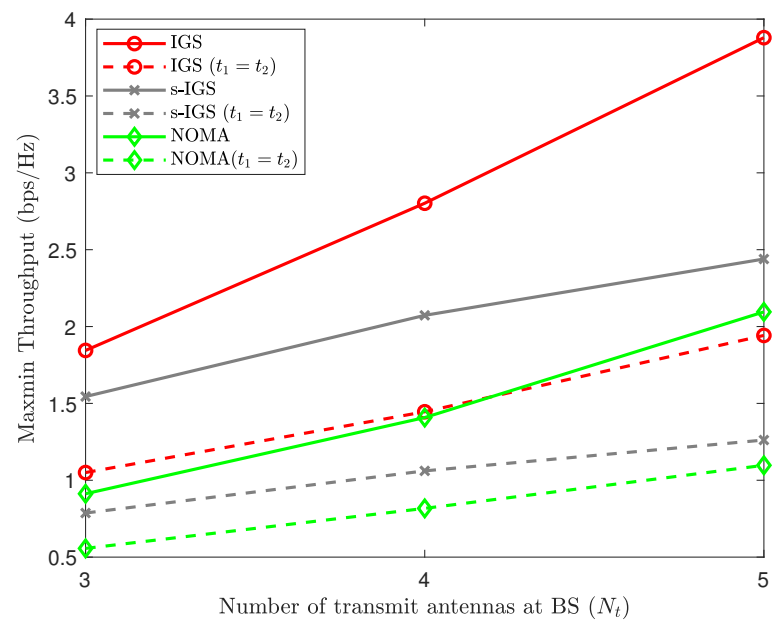

(a)

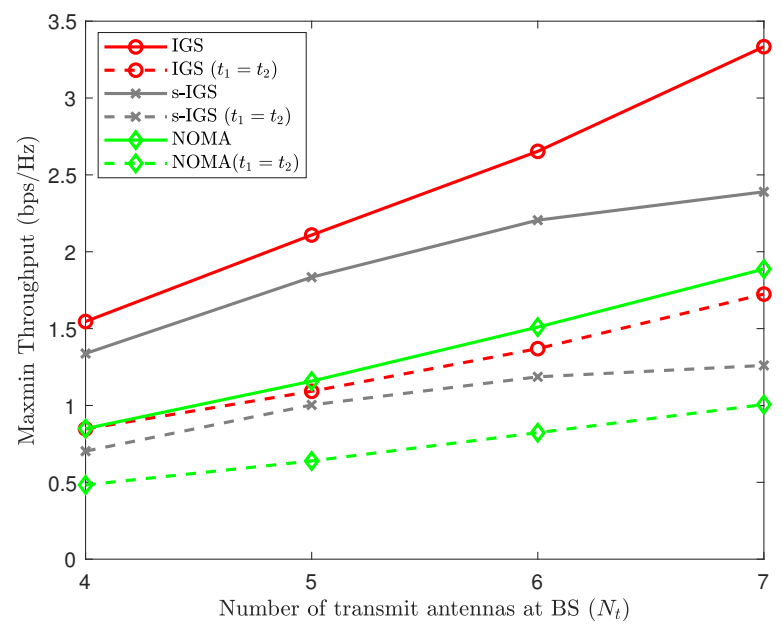

(b)

Fig. 8: Achievable minimum throughput vs BS antennas number $N_{t}:(a)(K, M)=(2,4) ;(b)(K, M)=(3,6)$

advantages of IGS over NOMA and OMA proper Gaussian signaling. IGS is most beneficial in the most vital lower power regime or when we have a low number of transmit antennas (compared to the number of users).

The exploitation of IGS in massive multi-input multi-output energy-harvesting enabled networks is under current study.

\section{APPENDIX: FUNDAMENTAL INEQUALITIES}

The following inequalities hold true for matrices of dimension $2 \times 2$ [39] and [40]:

$$
\begin{array}{r}
\ln \left|\mathbf{I}_{2}+[\mathbf{V}]^{2}(\mathbf{Y})^{-1}\right| \geq \\
\ln \left|\mathbf{I}_{2}+[\overline{\mathbf{V}}]^{2}(\overline{\mathbf{Y}})^{-1}\right| \\
-\left\langle[\overline{\mathbf{V}}]^{2}(\overline{\mathbf{Y}})^{-1}\right\rangle+2 \Re\left\{\left\langle\overline{\mathbf{V}}{ }^{H}(\overline{\mathbf{Y}})^{-1} \mathbf{V}\right\rangle\right\} \\
-\left\langle(\overline{\mathbf{Y}})^{-1}-\left(\overline{\mathbf{Y}}+[\overline{\mathbf{V}}]^{2}\right)^{-1},[\mathbf{V}]^{2}+\mathbf{Y}\right\rangle, \\
\forall \mathbf{V}, \mathbf{X} \succeq 0, \mathbf{Y} \succ 0 \quad \& \quad \overline{\mathbf{V}}, \overline{\mathbf{X}} \succeq 0, \overline{\mathbf{Y}} \succ 0,
\end{array}
$$

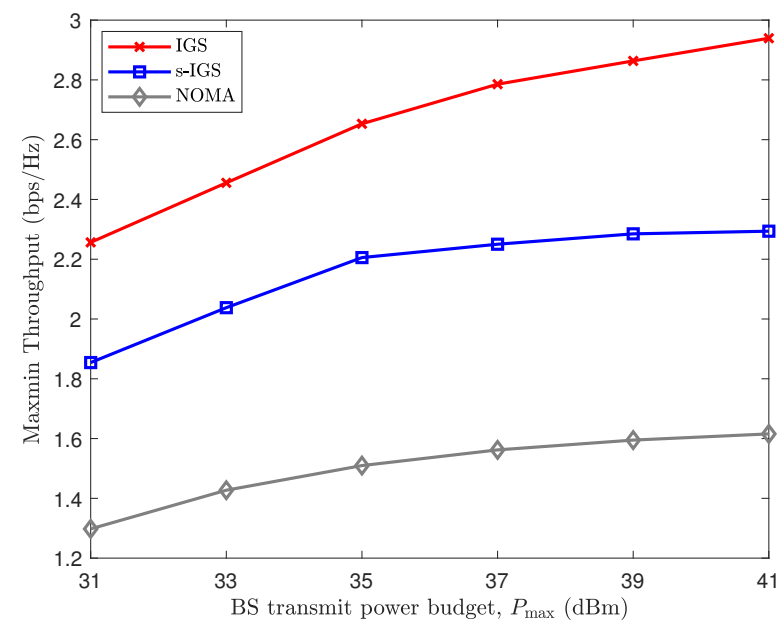

Fig. 9: Achievable minimum throughput vs BS transmit power budget $P$

and

$$
\begin{array}{r}
\ln \left|\mathbf{I}_{2}+\mathbf{X}(\mathbf{Y})^{-1}\right| \geq \\
\ln \left|\mathbf{I}_{2}+\overline{\mathbf{X}}(\overline{\mathbf{Y}})^{-1}\right|+4 \\
-\left\langle\overline{\mathbf{X}}+\overline{\mathbf{Y}},(\mathbf{X}+\mathbf{Y})^{-1}\right\rangle-\left\langle(\overline{\mathbf{Y}})^{-1}, \mathbf{Y}\right\rangle \\
\forall \mathbf{V}, \mathbf{X} \succeq 0, \mathbf{Y} \succ 0 \quad \& \quad \overline{\mathbf{V}}, \overline{\mathbf{X}} \succeq 0, \overline{\mathbf{Y}} \succ 0,
\end{array}
$$

and

$$
\begin{array}{r}
\ln \left|\mathbf{X}^{-1}+\mathbf{Y}^{-1}\right| \geq \\
\log \left|\overline{\mathbf{X}}^{-1}+\overline{\mathbf{Y}}^{-1}\right|+2 \\
-\left\langle(\overline{\mathbf{X}})^{-1}-(\overline{\mathbf{X}}+\overline{\mathbf{Y}})^{-1}, \mathbf{X}\right\rangle \\
-\left\langle(\overline{\mathbf{Y}})^{-1}-(\overline{\mathbf{X}}+\overline{\mathbf{Y}})^{-1}, \mathbf{Y}\right\rangle
\end{array}
$$$$
\forall \mathbf{X} \succ 0, \mathbf{Y} \succ 0 \quad \& \quad \overline{\mathbf{X}} \succ 0, \overline{\mathbf{Y}} \succ 0
$$

and

$$
\begin{array}{r}
\log |\mathbf{X}| \geq \log |\overline{\mathbf{X}}|+2-\left\langle\overline{\mathbf{X}},(\mathbf{X})^{-1}\right\rangle \\
\forall \mathbf{X} \succ 0 \quad \& \quad \overline{\mathbf{X}} \succ 0 .
\end{array}
$$

Theorem 1: The following inequalities hold true for all $\mathbf{X} \succ 0, \mathbf{Y} \succ 0$ and $\overline{\mathbf{X}} \succ 0, \overline{\mathbf{Y}} \succ 0$

$$
\langle\mathbf{X}, \mathbf{Y}\rangle \leq \frac{1}{4}\left\|\overline{\mathbf{X}}^{-1 / 2} \mathbf{X} \overline{\mathbf{Y}}^{1 / 2}+\overline{\mathbf{X}}^{1 / 2} \mathbf{Y} \overline{\mathbf{Y}}^{-1 / 2}\right\|^{2}
$$

and

$$
\langle\mathbf{X}, \mathbf{Y}\rangle \leq \frac{1}{2}\left(\left\|\overline{\mathbf{X}}^{-1 / 2} \mathbf{X} \overline{\mathbf{Y}}^{1 / 2}\right\|^{2}+\left\|\overline{\mathbf{X}}^{1 / 2} \mathbf{Y} \overline{\mathbf{Y}}^{-1 / 2}\right\|^{2}\right)
$$

Proof It follows from the matrix inequality

$$
\begin{aligned}
0 \preceq & \left(\overline{\mathbf{X}}^{-1 / 2} \mathbf{X} \overline{\mathbf{Y}}^{1 / 2}-\overline{\mathbf{X}}^{1 / 2} \mathbf{Y} \overline{\mathbf{Y}}^{-1 / 2}\right) \\
& \times\left(\overline{\mathbf{X}}^{-1 / 2} \mathbf{X} \overline{\mathbf{Y}}^{1 / 2}-\overline{\mathbf{X}}^{1 / 2} \mathbf{Y} \overline{\mathbf{Y}}^{-1 / 2}\right)^{H} \\
= & \left(\overline{\mathbf{X}}^{-1 / 2} \mathbf{X} \overline{\mathbf{Y}}^{1 / 2}+\overline{\mathbf{X}}^{1 / 2} \mathbf{Y} \overline{\mathbf{Y}}^{-1 / 2}\right) \\
& \times\left(\overline{\mathbf{X}}^{-1 / 2} \mathbf{X} \overline{\mathbf{Y}}^{1 / 2}+\overline{\mathbf{X}}^{1 / 2} \mathbf{Y} \overline{\mathbf{Y}}^{-1 / 2}\right)^{H} \\
& -2\left(\overline{\mathbf{X}}^{-1 / 2} \mathbf{X} \overline{\mathbf{Y}}^{1 / 2} \overline{\mathbf{Y}}^{-1 / 2} \mathbf{Y} \overline{\mathbf{X}}^{1 / 2}\right. \\
& \left.+\overline{\mathbf{X}}^{1 / 2} \mathbf{Y} \overline{\mathbf{Y}}^{-1 / 2} \overline{\mathbf{Y}}^{1 / 2} \mathbf{X} \overline{\mathbf{X}}^{-1 / 2}\right) \\
= & \left(\overline{\mathbf{X}}^{-1 / 2} \mathbf{X} \overline{\mathbf{Y}}^{1 / 2}+\overline{\mathbf{X}}^{1 / 2} \mathbf{Y} \overline{\mathbf{Y}}^{-1 / 2}\right) \\
& \times\left(\overline{\mathbf{X}}^{-1 / 2} \mathbf{X} \overline{\mathbf{Y}}^{1 / 2}+\overline{\mathbf{X}}^{1 / 2} \mathbf{Y} \overline{\mathbf{Y}}^{-1 / 2}\right)^{H} \\
& -2\left(\overline{\mathbf{X}}^{-1 / 2} \mathbf{X} \mathbf{Y} \overline{\mathbf{X}}^{1 / 2}+\overline{\mathbf{X}}^{1 / 2} \mathbf{Y} \mathbf{X} \overline{\mathbf{X}}^{-1 / 2}\right)
\end{aligned}
$$


that

$$
\begin{aligned}
& \overline{\mathbf{X}}^{-1 / 2} \mathbf{X} \mathbf{Y} \overline{\mathbf{X}}^{1 / 2}+\overline{\mathbf{X}}^{1 / 2} \mathbf{Y} \mathbf{X} \overline{\mathbf{X}}^{-1 / 2} \\
& \frac{1}{2}\left(\overline{\mathbf{X}}^{-1 / 2} \mathbf{X} \overline{\mathbf{Y}}^{1 / 2}+\overline{\mathbf{X}}^{1 / 2} \mathbf{Y} \overline{\mathbf{Y}}^{-1 / 2}\right) \\
& \times\left(\overline{\mathbf{X}}^{-1 / 2} \mathbf{X} \overline{\mathbf{Y}}^{1 / 2}+\overline{\mathbf{X}}^{1 / 2} \mathbf{Y} \overline{\mathbf{Y}}^{-1 / 2}\right)^{H} .
\end{aligned}
$$

Therefore

$$
\begin{aligned}
2\langle\mathbf{X Y}\rangle & =\left\langle\overline{\mathbf{X}}^{-1 / 2} \mathbf{X Y} \overline{\mathbf{X}}^{1 / 2}\right\rangle+\left\langle\overline{\mathbf{X}}^{1 / 2} \mathbf{Y} \mathbf{X} \overline{\mathbf{X}}^{-1 / 2}\right\rangle \\
& \leq \frac{1}{2}\left\|\overline{\mathbf{X}}^{-1 / 2} \mathbf{X} \overline{\mathbf{Y}}^{1 / 2}+\overline{\mathbf{X}}^{1 / 2} \mathbf{Y} \overline{\mathbf{Y}}^{-1 / 2}\right\|^{2}
\end{aligned}
$$

which is (65).

Furthermore, we use

$$
\begin{aligned}
&\left(\overline{\mathbf{X}}^{-1 / 2} \mathbf{X} \overline{\mathbf{Y}}^{1 / 2}-\overline{\mathbf{X}}^{1 / 2} \mathbf{Y} \overline{\mathbf{Y}}^{-1 / 2}\right) \\
& \times\left(\overline{\mathbf{X}}^{-1 / 2} \mathbf{X} \overline{\mathbf{Y}}^{1 / 2}-\overline{\mathbf{X}}^{1 / 2} \mathbf{Y} \overline{\mathbf{Y}}^{-1 / 2}\right)^{H} \succeq 0
\end{aligned}
$$

that yields

$$
\begin{gathered}
\overline{\mathbf{X}}^{-1 / 2} \mathbf{X} \overline{\mathbf{Y}}^{1 / 2} \overline{\mathbf{Y}}^{-1 / 2} \mathbf{Y} \overline{\mathbf{X}}^{1 / 2}+\overline{\mathbf{X}}^{1 / 2} \mathbf{Y} \overline{\mathbf{Y}}^{-1 / 2} \overline{\mathbf{Y}}^{1 / 2} \mathbf{X} \overline{\mathbf{X}}^{-1 / 2} \\
\preceq \overline{\mathbf{X}}^{-1 / 2} \mathbf{X} \overline{\mathbf{Y}}^{1 / 2}\left(\overline{\mathbf{X}}^{-1 / 2} \mathbf{X} \overline{\mathbf{Y}}^{1 / 2}\right)^{H} \\
+\overline{\mathbf{X}}^{1 / 2} \mathbf{Y} \overline{\mathbf{Y}}^{-1 / 2}\left(\overline{\mathbf{X}}^{1 / 2} \mathbf{Y} \overline{\mathbf{Y}}^{-1 / 2}\right)^{H}
\end{gathered}
$$

Therefore,

$$
\begin{aligned}
2\langle\mathbf{X Y}\rangle= & \left\langle\overline{\mathbf{X}}^{-1 / 2} \mathbf{X} \overline{\mathbf{Y}}^{1 / 2} \overline{\mathbf{Y}}^{-1 / 2} \mathbf{Y} \overline{\mathbf{X}}^{1 / 2}\right\rangle \\
& +\left\langle\overline{\mathbf{X}}^{1 / 2} \mathbf{Y} \overline{\mathbf{Y}}^{-1 / 2} \overline{\mathbf{Y}}^{1 / 2} \mathbf{X} \overline{\mathbf{X}}^{-1 / 2}\right\rangle \\
\leq & \left\|\overline{\mathbf{X}}^{-1 / 2} \mathbf{X} \overline{\mathbf{Y}}^{1 / 2}\right\|^{2}+\left\|\overline{\mathbf{X}}^{1 / 2} \mathbf{Y} \overline{\mathbf{Y}}^{-1 / 2}\right\|^{2}
\end{aligned}
$$

which is (66).

\section{REFERENCES}

[1] J. Hu, K. Yang, G. Wen, and L. Hanzo, "Integrated data and energy communication network: A comprehensive survey," IEEE Commun. Surveys Tuts., vol. 20, no. 4, pp. 3169-3219, Fourthquarter 2018.

[2] I. Krikidis et al., "Simultaneous wireless information and power transfer in modern communications systems," IEEE Commun. Mag., vol. 52, no. 11, pp. 104-110, Nov. 2014

[3] R. Zhang, R. G. Maunder, and L. Hanzo, "Wireless information and power transfer: from scientific hypothesis to engineering practice," IEEE Commun. Mag., vol. 2015, no. 8, pp. 99-105, Aug. 2015.

[4] X. Lu, P. Wang, D. Niyato, D. I. Kim, and Z. Han, "Wireless networks with RF energy harvesting: A contemporary survey," IEEE Commun. Surveys Tuts., vol. 17, pp. 757-789, 2015.

[5] A. A. Nasir, X. Zhou, S. Durrani, and R. A. Kennedy, "Relaying protocols for wireless energy harvesting and information process." IEEE Trans. Wirel. Commun., vol. 12, no. 7, pp. 3622-3636, Jul. 2013.

[6] L. Zhao and X. Wang, "Massive MIMO downlink for wireless information and energy transfer with energy harvesting receivers," IEEE Trans. Commun., vol. 67, no. 5, pp. 3309-3322, May 2019.

[7] H. H. M. Tam, H. D. Tuan, A. A. Nasir, T. Q. Duong, and H. V. Poor, "MIMO energy harvesting in full-duplex multi-user networks," IEEE Trans. Wirel. Commun., vol. 16, no. 5, pp. 3282-3297, May 2017.

[8] A. A. Nasir, H. D. Tuan, T. Q. Duong, and H. V. Poor, "Secrecy rate beamforming for multicell networks with information and energy harvesting," IEEE Trans. Signal Process., vol. 65, no. 3, pp. 677-689, 2017.

[9] A. A. Nasir, H. D. Tuan, D. T. Ngo, T. Q. Duong, and H. V. Poor, "Beamforming design for wireless information and power transfer systems: Receive power-splitting versus transmit time-switching," IEEE Trans. Commun., vol. 65, no. 2, pp. 876-889, Feb. 2017.

[10] H. Sun, F. Zhou, R. Q. Hu, and L. Hanzo, "Robust beamforming design in a NOMA cognitive radio network relying on SWIPT," IEEE J. Sel. Areas Commun., vol. 37, no. 1, pp. 142-155, Jan 2019.

[11] A. A. Nasir, H. D. Tuan, T. Q. Duong, and M. Debbah, "NOMA throughput and energy efficiency in energy harvesting enabled networks," IEEE Trans. Commun., vol. 67, no. 9, pp. 6499-6511, Sept. 2019.

[12] I. E. Telatar, "Capacity of multi-antenna Gaussian channels," Eur. Trans. Telecommun., vol. 10, no. 6, pp. 585-595, Nov./Dec. 1999.

[13] P. J. Schreier and L. L. Scharf, Statistical Signal Processing of ComplexValued Data: The Theory of Improper and Noncircular Signals. Cambridge University Press, 2010.
[14] S. Javed, O. Amin, B. Shihada, and M.-S. Alouini, "A journey from improper Gaussian signaling to asymmetric signaling," 2019. [Online]. Available: https://www.researchgate.net/publication/334894860_A_Journey_ from_Improper_Gaussian_Signaling_to_Asymmetric_Signaling

[15] Y. Zeng, R. Zhang, E. Gunawan, and Y. L. Guan, "Optimized transmission with improper Gaussian signaling in the k-user MISO interference channel," IEEE Trans. Wirel. Commun., vol. 12, no. 12, pp. 6303-6313, Dec. 2013.

[16] Y. Zeng, C. M. Yetis, E. Gunawan, Y. L. Guan, and R. Zhang, “Transmit optimization with improper Gaussian signaling for interference channels," IEEE Trans. Signal Process., vol. 61, no. 11, pp. 2899-2913, Jun. 2013.

[17] Z. K. M. Ho and E. Jorswieck, "Improper Gaussian signaling on the two-user SISO interference channel," IEEE Trans. Wireless Commun., vol. 11, no. 9, pp. 3194-3203, Sep. 2012.

[18] C. Lameiro and I. Santamara, "Degrees-of-freedom for the 4-user SISO interference channel with improper signaling," in Proc IEEE Inter. Conf. Commun. (ICC), Jun. 2013, pp. 3053-3057.

[19] H. D. Nguyen, R. Zhang, and S. Sun, "Improper signaling for symbol error rate minimization in k-user interference channel," IEEE Trans. Commun., vol. 63, no. 3, pp. 857-869, Mar. 2015.

[20] C. Hellings and W. Utschick, "Improper signaling versus time-sharing in the SISO Z-interference channel," IEEE Communications Letters, vol. 21, no. 11, pp. 2432-2435, Nov 2017.

[21] E. Kurniawan and S. Sun, "Improper Gaussian signaling scheme for the z-interference channel," IEEE Trans. Wirel. Commun., vol. 14, no. 7, pp. 3912-3923, Jul. 2015.

[22] C. Lameiro, I. Santamara, and P. J. Schreier, "Rate region boundary of the SISO Z-interference channel with improper signaling," IEEE Trans. on Commun., vol. 65, no. 3, pp. 1022-1034, Mar. 2017.

[23] V. R. Cadambe, S. A. Jafar, and C. Wang, "Interference alignment with asymmetric complex signalingsettling the Host-Madsen-Nosratinia conjecture," IEEE Trans. Inf. Theory, vol. 56, no. 9, pp. 4552-4565, Sept 2010.

[24] S. A. Jafar, "Interference alignment a new look at signal dimensions in a communication network," Foundations and Trends in Communications and Information Theory, vol. 7, no. 1, pp. 1-134, 2011. [Online]. Available: http://dx.doi.org/10.1561/0100000047

[25] L. Yang and W. Zhang, "Interference alignment with asymmetric complex signaling on MIMO X channels," IEEE Trans. Commun., vol. 62, no. 10, pp. 3560-3570, Oct 2014.

[26] S. Lagen, A. Agustin, and J. Vidal, "Coexisting linear and widely linear transceivers in the MIMO interference channel," IEEE Trans. Signal Process., vol. 64, no. 3, pp. 652-664, Feb 2016.

[27] — " "On the superiority of improper Gaussian signaling in wireless interference MIMO scenarios," IEEE Trans. Commun., vol. 64, no. 8, pp. 3350-3368, Aug. 2016.

[28] H. Shin, S. Park, H. Park, and I. Lee, "A new approach of interference alignment through asymmetric complex signaling and multiuser diversity," IEEE Trans. on Wirel. Commun., vol. 11, no. 3, pp. 880-884, Mar. 2012.

[29] C. Hellings, M. Joham, and W. Utschick, "QoS feasibility in MIMO broadcast channels with widely linear transceivers," IEEE Signal Process. Letts., vol. 20, no. 11, pp. 1134-1137, Nov. 2013.

[30] A. A. Nasir, H. D. Tuan, T. Q. Duong, and H. V. Poor, "Improper Gaussian signaling for broadcast interference networks," IEEE Signal Process. Lett., vol. 26, no. 6, pp. 808-812, Jun. 2019.

[31] C. Lameiro, I. Santamaria, and P. J. Schreier, "Benefits of improper signaling for underlay cognitive radio," IEEE Commun. Letts., vol. 4, no. 1, pp. 22-25, Feb. 2015.

[32] O. Amin, W. Abediseid, and M.-S. Alouini, "Underlay cognitive radio systems with improper Gaussian signaling: outage performance analysis," IEEE Trans. Wirel. Commun., vol. 15, no. 7, pp. 4875-4887, Jul. 2016.

[33] C. Lameiro, I. Santamaria, and P. J. Schreier, "Improper Gaussian signaling for multiple-access channels in underlay cognitive radio," IEEE Trans. Commun., vol. 67, no. 3, pp. 1817-1830, Mar. 2019.

[34] H. D. Tuan, A. A. Nasir, H. H. Nguyen, T. Q. Duong, and H. V. Poor, "Non-orthogonal multiple access with improper Gaussian signaling," IEEE J. Selec. Topics Signal Process., vol. 13, no. 3, pp. 496-507, Mar. 2019.

[35] T. M. Cover and J. A. Thomas, Elements of Information Theory (second edition). John Wileys \& Sons, 2006.

[36] H. Tuy, Convex Analysis and Global Optimization (second edition). Springer International, 2016. 
[37] D. Peaucelle, D. Henrion, and Y. Labit, "Users guide for SeDuMi interface 1.03," 2002. [Online]. Available: http://homepages.laas.fr/ peaucell/software/sdmguide.pdf

[38] B. R. Marks and G. P. Wright, "A general inner approximation algorithm for nonconvex mathematical programms," Operations Research, vol. 26, no. 4, pp. 681-683, Jul. 1978.

[39] H. H. M. Tam, H. D. Tuan, and D. T. Ngo, "Successive convex quadratic programming for quality-of-service management in full-duplex MUMIMO multicell networks," IEEE Trans. Commun., vol. 64, no. 6, pp. 2340-2353, Jun. 2016.

[40] L. D. Nguyen, H. D. Tuan, T. Q. Duong, and H. V. Poor, "Multi-user regularized zero-forcing beamforming," IEEE Trans. Signal Process., vol. 67 , no. 11 , pp. 2839-2853, Jun. 2019.

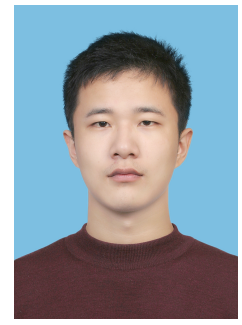

Hongwen Yu was born in Jingdezhen, China. He received the B.S. degree in Communication and Information Engineering from the Shanghai University, Shanghai, China, in 2011, and the M.S. degree in Communication and Information Engineering from the Shanghai University, Shanghai, China, in 2014. $\mathrm{He}$ is currently pursuing the Ph.D. degree with the School of Electrical and Data Engineering, University of Technology Sydney, Ultimo, NSW, Australia and with Shanghai University, Shanghai, China. His current research interests include optimization methods for wireless communication and signal processing.

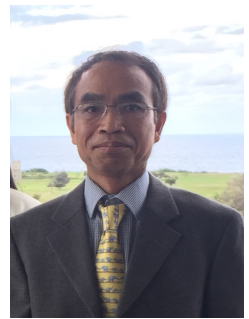

Hoang Duong Tuan received the Diploma (Hons.) and Ph.D. degrees in applied mathematics from Odessa State University, Ukraine, in 1987 and 1991, respectively. He spent nine academic years in Japan as an Assistant Professor in the Department of Electronic-Mechanical Engineering, Nagoya University, from 1994 to 1999, and then as an Associate Professor in the Department of Electrical and Computer Engineering, Toyota Technological Institute, Nagoya, from 1999 to 2003. He was a Professor with the School of Electrical Engineering and Telecommunications, University of New South Wales, from 2003 to 2011. $\mathrm{He}$ is currently a Professor with the School of Electrical and Data Engineering, University of Technology Sydney. He has been involved in research with the areas of optimization, control, signal processing, wireless communication, and biomedical engineering for more than 20 years.

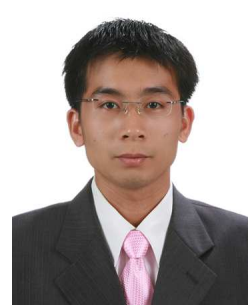

Trung Q. Duong (S'05, M'12, SM'13) received his Ph.D. degree in Telecommunications Systems from Blekinge Institute of Technology (BTH), Sweden in 2012. Currently, he is with Queen's University Belfast (UK), where he was a Lecturer (Assistant Professor) from 2013 to 2017 and a Reader (Associate Professor) from 2018. His current research interests include Internet of Things (IoT), wireless communications, molecular communications, and signal processing. He is the author or co-author of 290 technical papers published in scientific journals (165 articles) and presented at international conferences (125 papers).

Dr. Duong currently serves as an Editor for the IEEE TRANSACTIONS ON WIRELESS COMMUNICATIONS, IEEE TRANSACTIONS ON COMMUNICATIONS, IET COMmunications, and a Lead Senior Editor for IEEE Communications LetTers. He was awarded the Best Paper Award at the IEEE Vehicular Technology Conference (VTC-Spring) in 2013, IEEE International Conference on Communications (ICC) 2014, IEEE Global Communications Conference (GLOBECOM) 2016, and IEEE Digital Signal Processing Conference (DSP) 2017. He is the recipient of prestigious Royal Academy of Engineering Research Fellowship (2016-2021) and has won a prestigious Newton Prize 2017.
Yong Fang received the Ph.D. degree in Electronic Engineering from City University of Hong Kong, Hong Kong, in 1999. He is now a professor in the School of Communication and Information Engineering, Shanghai University, Shanghai, China. His research interests include communication signal processing, blind signal processing, and adaptive information system.

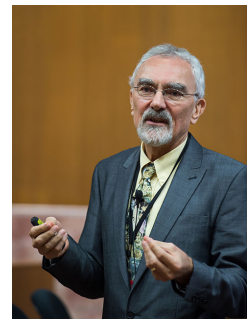

Lajos Hanzo ([http://www-mobile.ecs.soton.ac.uk, https://en.wikipedia.org/wiki/Lajos_Hanzo] http://www-mobile.ecs.soton.ac.uk, https://en.wikipedia.org/wiki/Lajos_Hanzo) FREng, FIEEE, FIET, Fellow of EURASIP, DSc holds an honorary doctorate by the Technical University of Budapest (2009) and by the University of Edinburgh (2015). He is a Foreign Member of the Hungarian Academy of Sciences and a former Editor-in-Chief of the IEEE Press. He has served as Governor of both IEEE ComSoc and of VTS. He has published 1900+ contributions at IEEE Xplore, 19 Wiley-IEEE Press books and has helped the fast-track career of $119 \mathrm{PhD}$ students. Over 40 of them are Professors at various stages of their careers in academia and many of them are leading scientists in the wireless industry. 